\title{
Rapid Identification of Characteristic Chemical Constituents of Panax ginseng, Panax quinquefolius, and Panax japonicus Using UPLC-Q-TOF/MS
}

\author{
Liu Jinbiao, ${ }^{1}$ Zhang Xinyue, ${ }^{2,3}$ Yang Shenshen $\mathbb{D}^{2},{ }^{2}$ Wang Shuo, ${ }^{2}$ Liu Chengcheng, ${ }^{2}$ \\ Yang Bin $(1),{ }^{2}$ Li Yubo $\mathbb{1},{ }^{2}$ and Cai Ting $\mathbb{1}^{3,4}$ \\ ${ }^{1}$ School of Chemistry and Chemical Engineering, Tianjin University of Technology, Tianjin 300384, China \\ ${ }^{2}$ Tianjin University of Traditional Chinese Medicine, No. 10 Poyang Lake Road, West Zone, Tuanbo New City, Jinghai District, \\ Tianjin 301617, China \\ ${ }^{3}$ Hwa Mei Hospital, University of Chinese Academy of Sciences (Ningbo No. 2 Hospital), Ningbo 315010, China \\ ${ }^{4}$ Ningbo Institute of Life and Health Industry, University of Chinese Academy of Sciences, Ningbo 315010, China
}

Correspondence should be addressed to Yang Bin; yang3023008@163.com, Li Yubo; yaowufenxi001@sina.com, and Cai Ting; caiting@ucas.ac.cn

Received 30 October 2021; Accepted 15 January 2022; Published 15 February 2022

Academic Editor: Eulogio J. Llorent Mart nez

Copyright (c) 2022 Liu Jinbiao et al. This is an open access article distributed under the Creative Commons Attribution License, which permits unrestricted use, distribution, and reproduction in any medium, provided the original work is properly cited.

\begin{abstract}
Saponins are the main active components in Panax ginseng C. A. Mey. (PG), Panax quinquefolius L. (PQ), and Panax japonicus C. A. Mey. (PJ), which belong to the genus Panax in the Araliaceae family. Because the chemical components in the three species are similar, they are often mixed and misused in functional foods and pharmaceuticals applications. Therefore, it is urgent to establish a method to quickly distinguish among PG, PQ, and PJ. Ultraperformance liquid chromatography quadrupole time-offlight mass spectrometry (UPLC-Q-TOF/MS) was combined with data postprocessing to identify the main characteristic fragments (CFs) and the related neutral losses (NLs) of protopanaxadiol (PPD), protopanaxatriol (PPT), oleanolic acid (OLE), and ocotillol- (OCO-) type saponins. By comparing the mass spectral data, it was possible to rapidly classify and identify saponins in PG, PQ, and PJ. A total of twenty-three chemical components were identified in the PG samples, twenty-three components were identified in the PQ samples, and twenty-seven components were identified in the PJ samples. Among them, OCO-type saponins were characteristic of PQ and PJ. Ginsenoside Rf, which was absent from PQ, allowed for differentiation between PQ and PJ. The CFs and NLs in the mass spectra of the characteristic components of PG, PQ, and PJ allowed for the rapid classification and identification of these species. Additionally, these results provide technical support for the quality evaluation of Chinese herbal medicine and for constructing a scientific regulatory system.
\end{abstract}

\section{Introduction}

Panax ginseng C. A. Mey. (PG), Panax quinquefolius L. (PQ), and Panax japonicus C. A. Mey. (PJ) are three important plants of the genus Panax in the Araliaceae family. Based on their morphology, these plants can be divided into two groups: the first is an upright rhizome with developed fleshy roots, mainly containing dammarane- (DAM-) type tetracyclic triterpenoid saponins, such as PG, PQ, Panax notoginseng, and so on. The other is a developed rhizome, horizontal bamboo whip or rosary, with less fleshy roots. It mainly contains oleanolic acid (OLE) pentacyclic triterpenoid saponins, such as PJ [1]. Recent investigations have shown that the main active components of PG, PQ, and PJ are saponins, polysaccharides, phenolic acids, and alkaloids. Recent pharmacological studies have shown that saponins can delay aging, improve immunity, prevent and treat Alzheimer's disease, and regulate the nervous system. Additionally, saponins exhibit antitumor activity, along with antioxidative, antihypertensive, and antihyperglycemic 
properties [2-6]. Consequently, ginsenosides are widely used in food, healthcare products, cosmetics, and medicine. Although the three traditional Chinese medicinal herbs from the genus Panax have different pharmacological actions, indications, and clinical applications, the properties and chemical composition of these Chinese herbal species are very similar, and thus adulterated products are often passed off as genuine in the market [7-10]. For example, in order to reduce the production cost or simply via mistaken identity, PQ is added to commercial PG products [11], and narrowleaf Panax japonicus and Panax notoginseng of the same or different families and genera are often used as adulterants intentionally or mistakenly as a substitute for genuine PJ [12]. Adulterants not only compromise the integrity of the Chinese herbal medicine market but also affect the efficacy and safety of traditional Chinese medicine. Therefore, it is urgent to establish methods for the rapid identification of the three genuses of Panax used in traditional Chinese medicines so as to improve the efficacy of quality evaluation and provide scientific regulation.

The ginsenosides found in PG can be divided into two groups according to their glycosidic structure: DAM-type and OLE-type. There are two types of DAM: protopanaxadiol(PPD-) type saponins, for which the aglycone is 20(s)-PPD; these contain the most ginsenosides, including ginsenoside $\mathrm{Rb}_{1}, \mathrm{Rb}_{2}, \mathrm{Rb}_{3}, \mathrm{Rc}, \mathrm{Rd}, \mathrm{Rg}_{3}$, and $\mathrm{Rh}_{2}$, and protopanaxatriol(PPT-) type saponins, for which the aglycone is 20(s)-PPT, including ginsenoside $\mathrm{Re}, \mathrm{Rf}, \mathrm{Rg}_{1}$, and $\mathrm{Rh}_{1}$. The aglycone of OLE-type ginsenosides, such as ginsenoside Ro, is oleanolic acid [13]. Compared to PG, PQ and PJ not only contain PPD-, PPT-, and OLE-type saponins but also contain ocotillol(OCO-) type saponins, such as pseudoginsenoside $\mathrm{F}_{11}$ and pseudoginsenoside $\mathrm{RT}_{4}[14,15]$. In addition, ginsenoside $\mathrm{Rf} \mathrm{has}$ not been found in PQ [16]. The types of saponins, similar to the structures of their parental nucleus, are rich and complex. Therefore, it is necessary to develop a rapid method for the qualitative analysis of saponins that allows for the accurate classification and identification of different traditional Chinese medicines from the genus Panax.

In this study, an accurate, rapid, and sensitive ultraperformance liquid chromatography quadrupole tandem timeof-flight mass spectrometry (UPLC-Q-TOF/MS) technique combined with data postprocessing is established (Figure 1). First, the characteristic fragments (CFs) and neutral losses (NLs) of various saponins are summarized. Based on the quasimolecular ions and the fragment ions provided by high-resolution mass spectrometry, the chromatographic retention time, and related literature data, the saponin profiles of PG, PQ, and PJ are identified in order to realize accurate distinction between the three. This study aims to explore the medicinal basis of the three traditional Chinese medicinal herbs from the genus Panax and provide basic information for establishing a comprehensive system for evaluating the quality of medicinal materials. Simultaneously, this approach can provide technical support for constructing a scientifically based regulatory system.

\section{Materials and Methods}

2.1. Materials, Reagents, and Instruments. Nine batches of representative medicinal materials were collected or purchased from Jilin, the main area producing PG and PQ, and from different areas producing PJ. The detailed sample information is presented in Table 1. High-performance liquid chromatography-grade acetonitrile was provided by Oceanpak (Sweden), high-performance liquid chromatography-grade formic acid was provided by Thermo Fisher (USA), and distilled water was purchased from Watsons Food and Beverage Company (China). A Waters Acquity (Waters, USA) UPLC instrument and a Xevo G2 (Waters, USA) Q-TOF/MS system were used in this study.

2.2. Sample Preparation. The Chinese medicinal herbs PG-1, PQ-1, and PJ-1 were, respectively, crushed, and $0.2 \mathrm{~g}$ of the powdered PG-1, PQ-1, and PJ-1 was placed into three separate test tubes, soaked in $10 \mathrm{~mL}$ of $70 \%$ ethanol, and ultrasonically extracted for $50 \mathrm{~min}$. After extraction, each tube was cooled and centrifuged for $10 \mathrm{~min}$. The supernatant was subsequently filtered through a $0.22 \mu \mathrm{m}$ microporous membrane and analyzed by UPLC-Q-TOF/MS.

2.3. UPLC and MS Conditions. UPLC conditions were as follows: a Waters Acquity UPLC BEH C18 column $(2.1 \mathrm{~mm} \times 100 \mathrm{~mm}, 1.7 \mu \mathrm{m})$ was used as the chromatographic column. The column temperature was set at $40^{\circ} \mathrm{C}$, the flow rate was $0.3 \mathrm{~mL} / \mathrm{min}$, the injection volume was $5 \mu \mathrm{L}$, the mobile phase was composed of $0.1 \%$ formic acid aqueous solution (A) and acetonitrile (B), and the chromatographic separation was carried out by gradient elution, where the gradient sequence was as follows: $0-2 \mathrm{~min}, 5-10 \% \mathrm{~B}$; 2-6 min, $10-30 \% \mathrm{~B} ; 6-10 \mathrm{~min}, 30-50 \% \mathrm{~B} ; 10-15 \mathrm{~min}$, $50-80 \%$ B; $15-20 \mathrm{~min}, 80-100 \%$ B; $20-25 \mathrm{~min}, 100 \% \mathrm{~B}$; 25-30 $\mathrm{min}, 100-5 \% \mathrm{~B}$; and $30-35 \mathrm{~min}, 5 \% \mathrm{~B}$.

TOF-MS conditions were as follows: mass spectrometry was performed using a Waters G2 Q-TOF mass spectrometer, equipped with a negative mode electrospray ionization source. The capillary voltage was $-2.4 \mathrm{kV}$, the cone voltage was $40 \mathrm{~V}$, the source temperature was $120^{\circ} \mathrm{C}$, the desolvation temperature was $400^{\circ} \mathrm{C}$, the desolvation gas was $800 \mathrm{~L} / \mathrm{h}$, and the cone gas was $50 \mathrm{~L} / \mathrm{h}$, using leucine enkephalin $(m / z 554.2615)$ as an external reference. In order to ensure the accuracy of the data acquisition, the full-scan data in the range of 100-1500 Da were obtained.

2.4. Method Establishment. The main pharmacological constituents of PG, PQ, and PJ are saponins. Therefore, to accurately distinguish the three traditional Chinese medicines, it was necessary to classify and identify the saponins. However, the use of conventional methods to determine the composition of saponins is complicated and time-consuming because of their large molecular weight and similar 


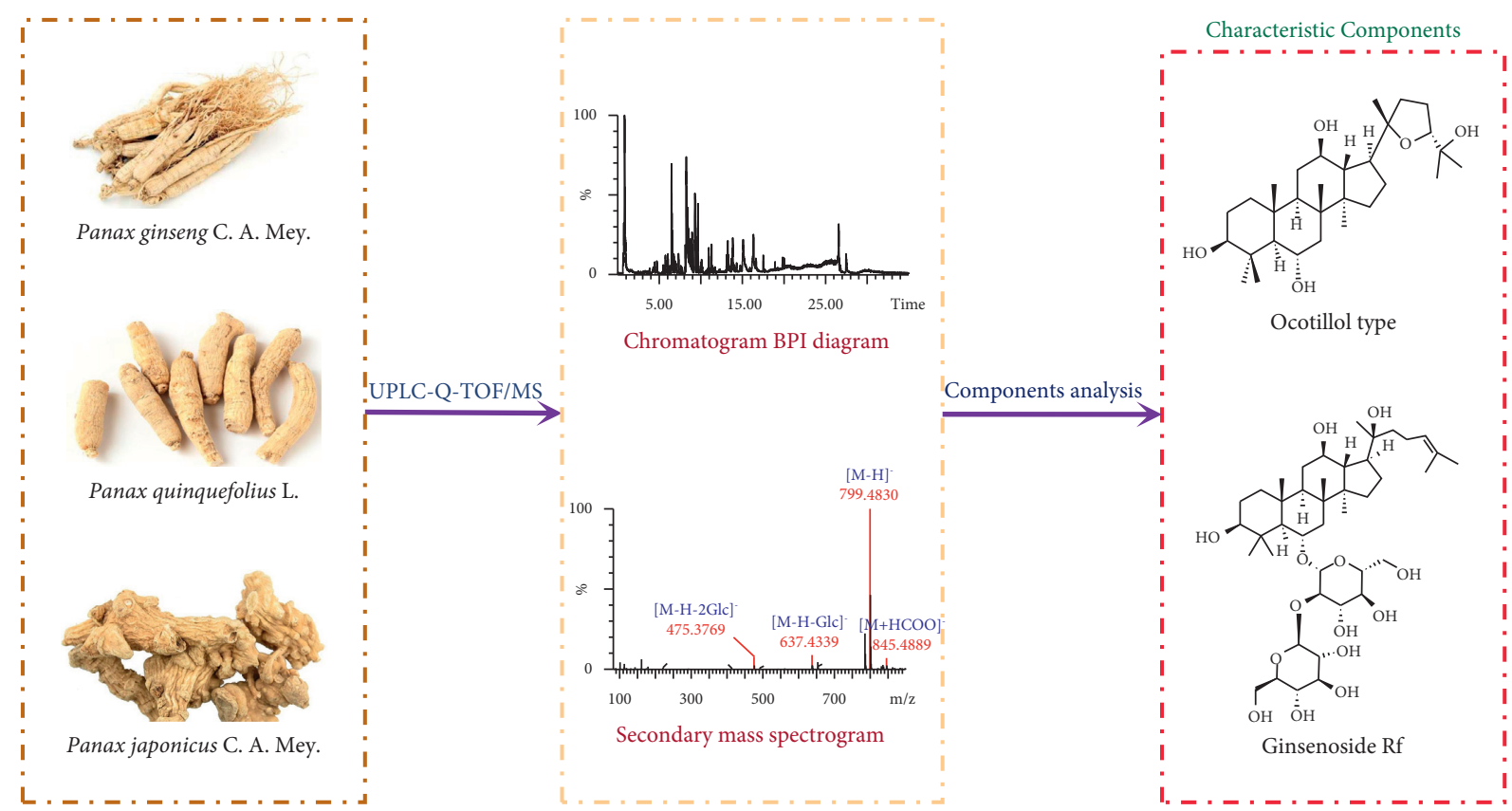

Figure 1: The rapid identification strategy of three traditional Chinese medicines in the genus Panax.

TABLE 1: Detailed information of the tested PG, PQ, and PJ samples.

\begin{tabular}{|c|c|c|}
\hline $\begin{array}{l}\text { Sample } \\
\text { number }\end{array}$ & Source & Identity \\
\hline PG-1 & Jilin province, China & Panax ginseng C. A. Mey. \\
\hline PG-2 & Jilin province, China & Panax ginseng C. A. Mey. \\
\hline PG-3 & Jilin province, China & Panax ginseng C. A. Mey. \\
\hline PQ-1 & Jilin province, China & Panax quinquefolius L. \\
\hline PQ-2 & Jilin province, China & Panax quinquefolius L. \\
\hline PQ-3 & Jilin province, China & Panax quinquefolius L. \\
\hline PJ-1 & $\begin{array}{c}\text { Anhui province, } \\
\text { China }\end{array}$ & $\begin{array}{c}\text { Panax japonicus C. A. } \\
\text { Mey. }\end{array}$ \\
\hline PJ-2 & $\begin{array}{c}\text { Sichuan province, } \\
\text { China }\end{array}$ & $\begin{array}{c}\text { Panax japonicus C. A. } \\
\text { Mey. }\end{array}$ \\
\hline PJ-3 & $\begin{array}{c}\text { Yunnan province, } \\
\text { China }\end{array}$ & $\begin{array}{c}\text { Panax japonicus C. A. } \\
\text { Mey. }\end{array}$ \\
\hline
\end{tabular}

core structure. In collision-induced MS, compounds with the same or similar parent nuclear skeletons usually fracture similarly, and this technique is used to establish fragmentation patterns. CFs are molecular compounds with the same or similar parent core structures. When exposed to the energy impact of MS, they can fragment into ions, from which the cleavage type and material can be easily inferred. CFs can be used to help to rapidly classify the target materials. In addition, molecular ions can lose neutral radicals or molecules in MS, as shown by the difference between the mass/load ratio and the molecular ion peak and the product ion peaks, respectively. These lost free-radicals or molecules are known as NLs, which aid the screening and identification of substances [17-22]. Therefore, we present the MS fragmentation of PG, PQ, and PJ and summarize their CFs and common NLs, which are based on the different core structures (DAM-, OLE-, and OCO-types). First, the different CFs were used to preliminarily classify the unknown components. The various saponins were identified by combined analysis of their molecular ions, retention time, and the fragmentation pattern of the unknown components, along with their fracture processes, which were estimated using common NLs. Based on the types of saponins in the samples, the three traditional Chinese medicinal herbs could be identified quickly and accurately.

\section{Results and Discussion}

Based on the summarized CF and NL data, PG, PQ, and PJ were analyzed. Twenty-three chemical constituents were identified for the PG samples, which included 10 PPD saponins, 11 PPT saponins, and 2 OLE saponins. A total of twenty-three components was identified from PQ, which included 12 PPD saponins, 4 PPT saponins, 3 OLE saponins, and 4 OCO saponins. A total of twenty-seven components was identified in the PJ samples, which included 7 PPD saponins, 6 PPT saponins, 11 OLE saponins, and 3 OCO saponins. The CFs and NLs of the different types of saponins are shown in Figure 2. The total ion chromatograms of the PG, PQ, and PJ extracts in negative ion mode are shown in Figure 3, and their compositions are shown in Tables 2-4.

\subsection{Analysis of Dammarane-Type Saponins by MS}

3.1.1. PPD-Type Saponins. PPD-type ginsenosides, such as ginsenosides $\mathrm{Rb}, \mathrm{Rb}_{2}, \mathrm{Rc}$, and $\mathrm{Rg}_{3}$, are saponins in the genus Panax. In 1966, Shibata et al. isolated ginsenediol from the root of ginseng for the first time and reported its chemical properties and structure [35]. Considering the structural types of PPD and the mass spectral information in the literature, it was found that two $\mathrm{CFs}$ were produced, with signals at $m / z 621\left[\mathrm{C}_{36} \mathrm{H}_{61} \mathrm{O}_{8}\right]^{-}$and $m / z 459\left[\mathrm{C}_{30} \mathrm{H}_{51} \mathrm{O}_{3}\right]^{-}$. At the same time, the product ions observed in the $\mathrm{MS}^{2}$ profiles 


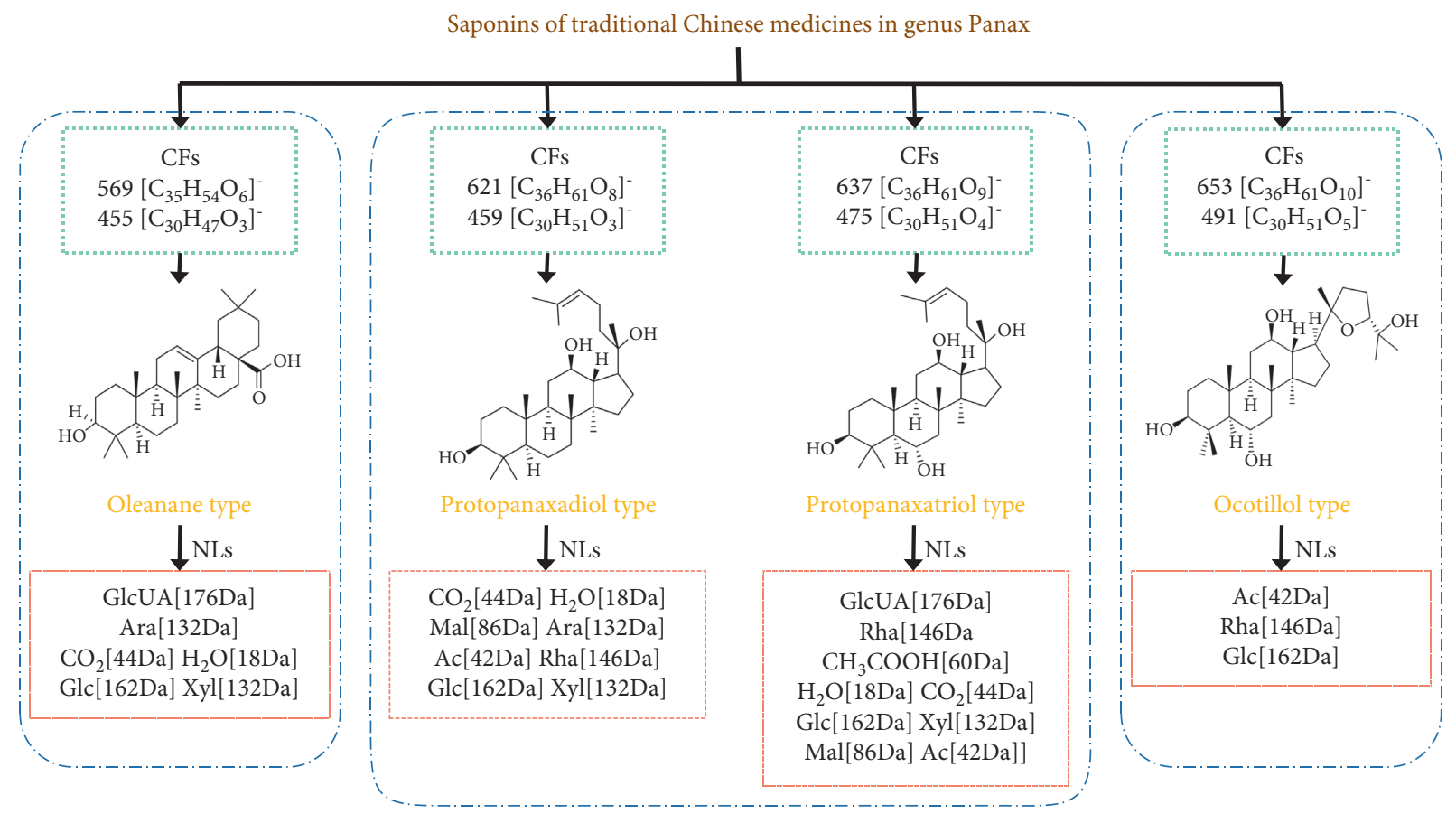

Figure 2: Characteristic fragments and neutral losses of different types of saponins in genus Panax. Ac: acetyl; Mal: malonyl; Glc: glucose residue; Ara: arabinose residue; Rha: rhamnose residue; Xyl: xylose residue; GlcUA: glucuronic acid.

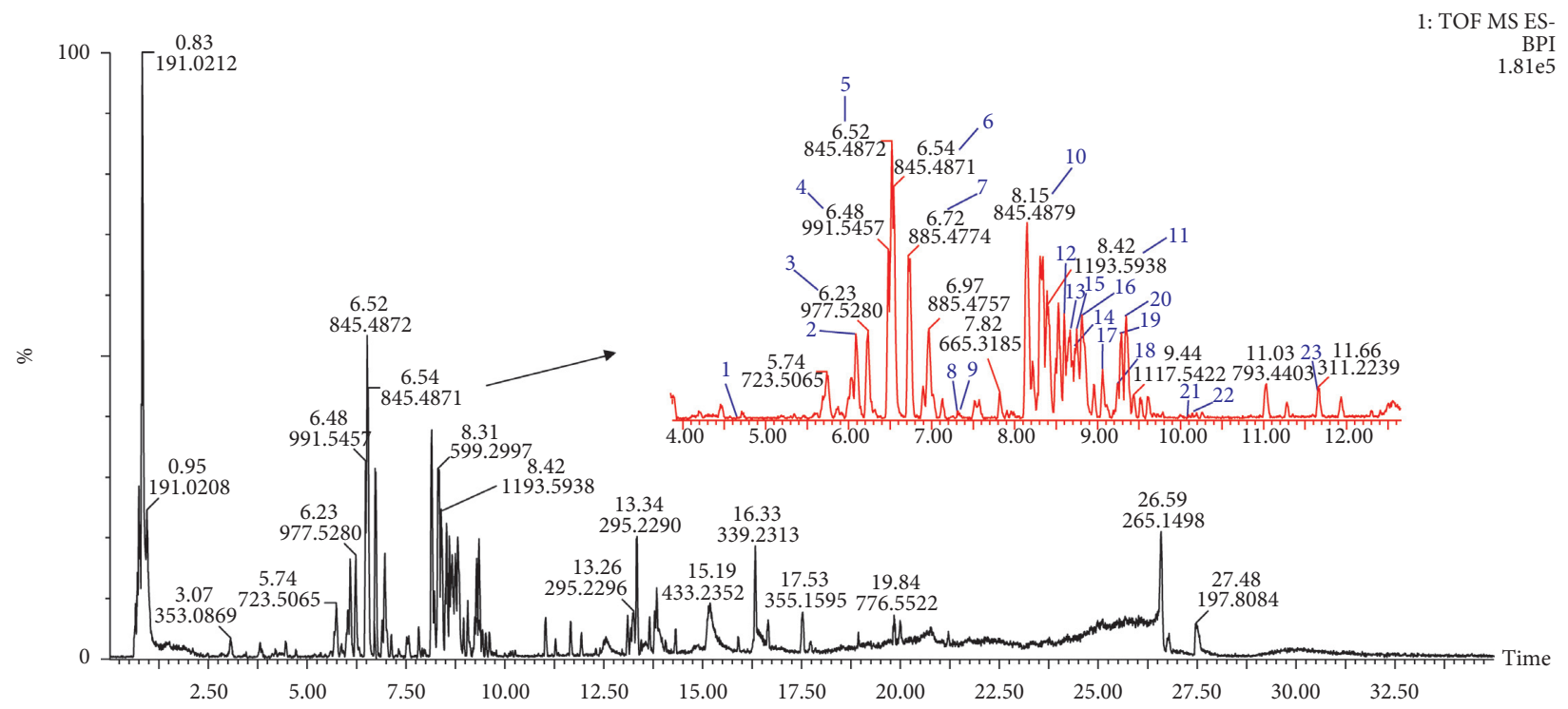

(a)

FIGURE 3: Continued. 


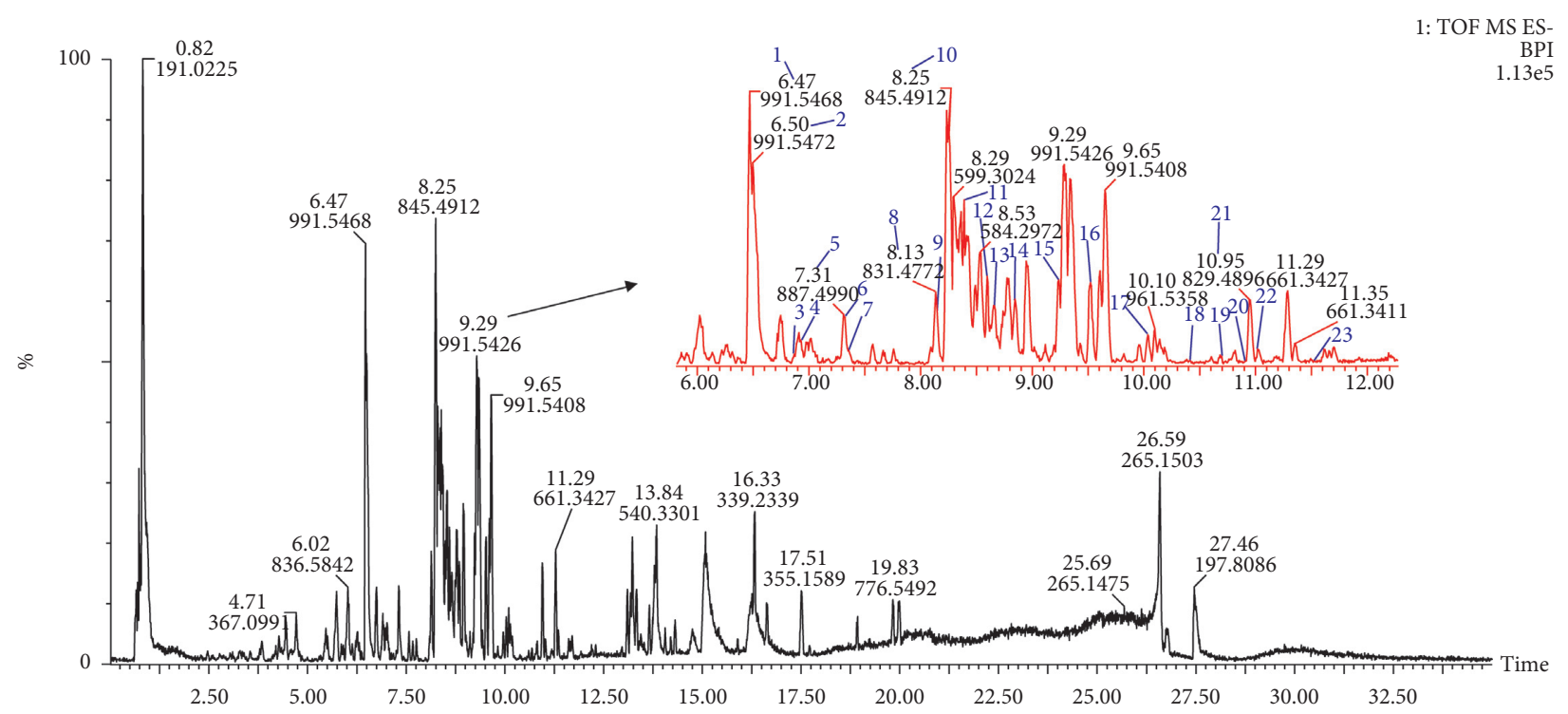

(b)

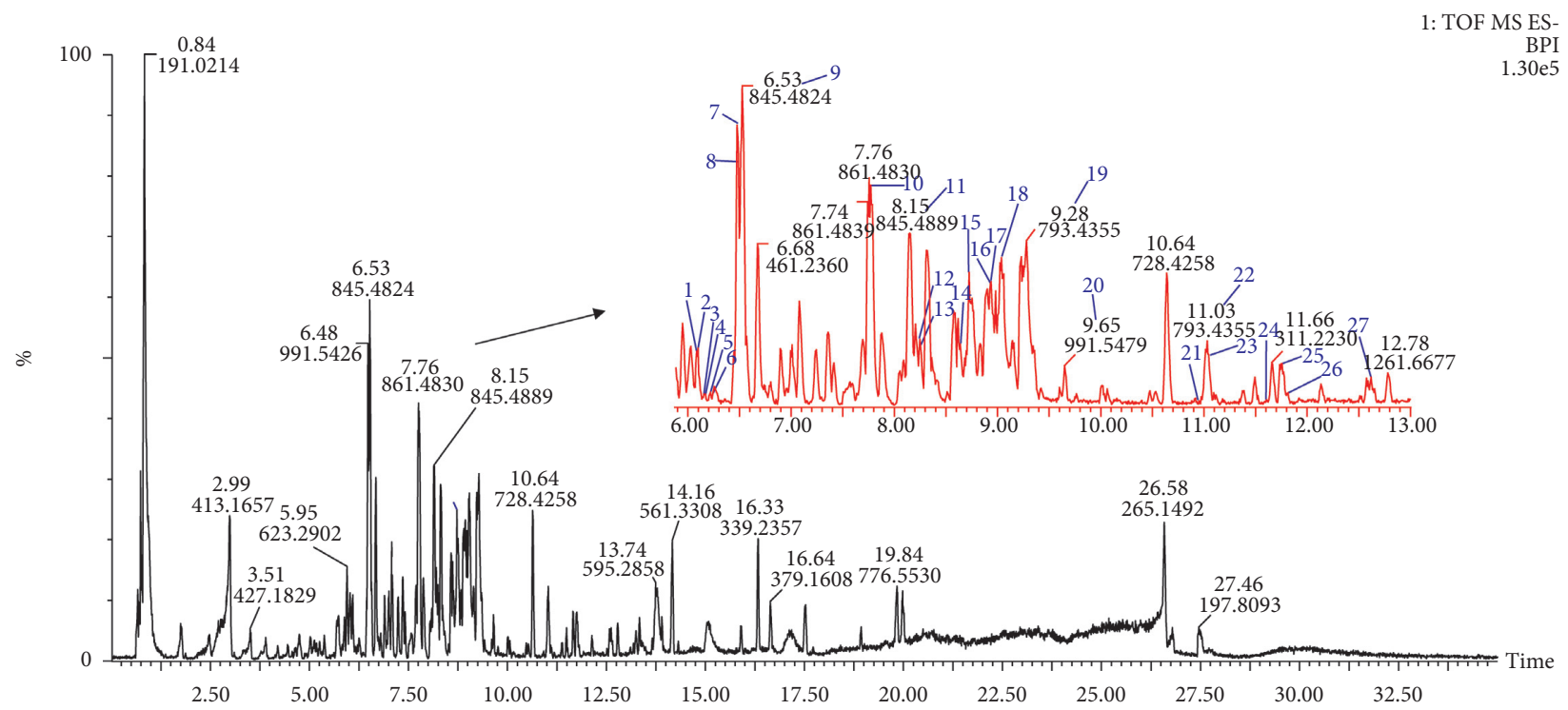

(c)

Figure 3: Chromatogram BPI diagram of PG, PJ, and PQ under negative ions (a) PG, (b) PQ, and (c) PJ.

of the PPD-type saponins generally resulted in the following NLs: $\mathrm{CO}_{2}(44 \mathrm{Da}), \mathrm{H}_{2} \mathrm{O}(18 \mathrm{Da}), \mathrm{Mal}(86 \mathrm{Da})$, Ara (132 Da), Glc (162 Da), Xyl (132 Da), Ac (42 Da), and Rha (146 Da). Therefore, based on the CFs and NLs, it was possible to identify the compounds and infer their fracture processes.

Compound 11 (Table 2) had a retention time of $8.42 \mathrm{~min}$ and a molecular formula of $\mathrm{C}_{57} \mathrm{H}_{94} \mathrm{O}_{26}$. In the negative ion mode, compound 11 produced a precursor ion at $\mathrm{m} / z$ $1193.5938[\mathrm{M}-\mathrm{H}]^{-}$and seven fragment ion peaks at $\mathrm{m} / z$ 1149.6027, 1107.5938, 945.5364, 783.4828, 765.4836, 621.4205, and 459.3793. Based on the CF ions at $\mathrm{m} / z$ 621.4205 and 459.3793, compound 11 in Table 2 could be preliminarily identified as a PPD-type saponin. The product ion at $m / z 1149.6027$ was produced by the removal of a $\mathrm{CO}_{2}$ molecule $(44 \mathrm{Da})$ from the precursor ion. The product ion at $m / z 1107.5938$ was produced by the malonyl group ( $86 \mathrm{Da}$ ) of the precursor ion. The $\mathrm{m} / z 945.5364$ product ion was produced by the neutral loss of malonyl and a part of the glucose residue $(162 \mathrm{Da})$ from the precursor ion. When the product ions at $\mathrm{m} / z 945.5364$ continued to lose glucose residues, product ions with $m / z 783.4828$ [M-H-Mal-2Glc] ${ }^{-}$, $\mathrm{m} / z$ 621.4205 [M-H-Mal-3Glc] ${ }^{-}$, and $\mathrm{m} / z$ 459.3793[M-HMal-4Glc $]^{-}$were formed. When the product ion with a peak at $m / z 783.4828$ lost one $\mathrm{H}_{2} \mathrm{O}$ molecule $(18 \mathrm{Da})$, the product ion at $\mathrm{m} / z 765.4836\left[\mathrm{M}-\mathrm{H}-\mathrm{Mal}-2 \mathrm{Glc}^{-} \mathrm{H}_{2} \mathrm{O}\right]^{-}$was formed. Therefore, compound 11 (Table 2) was identified as malonylginsenoside $\mathrm{Rb}_{1}$ from its molecular ion and secondary mass spectral fracture pattern $[24,30]$. The cleavage pathway of malonyl-ginsenoside $\mathrm{Rb}_{1}$ in negative ion mode is shown in Figures 4 and 5 . 


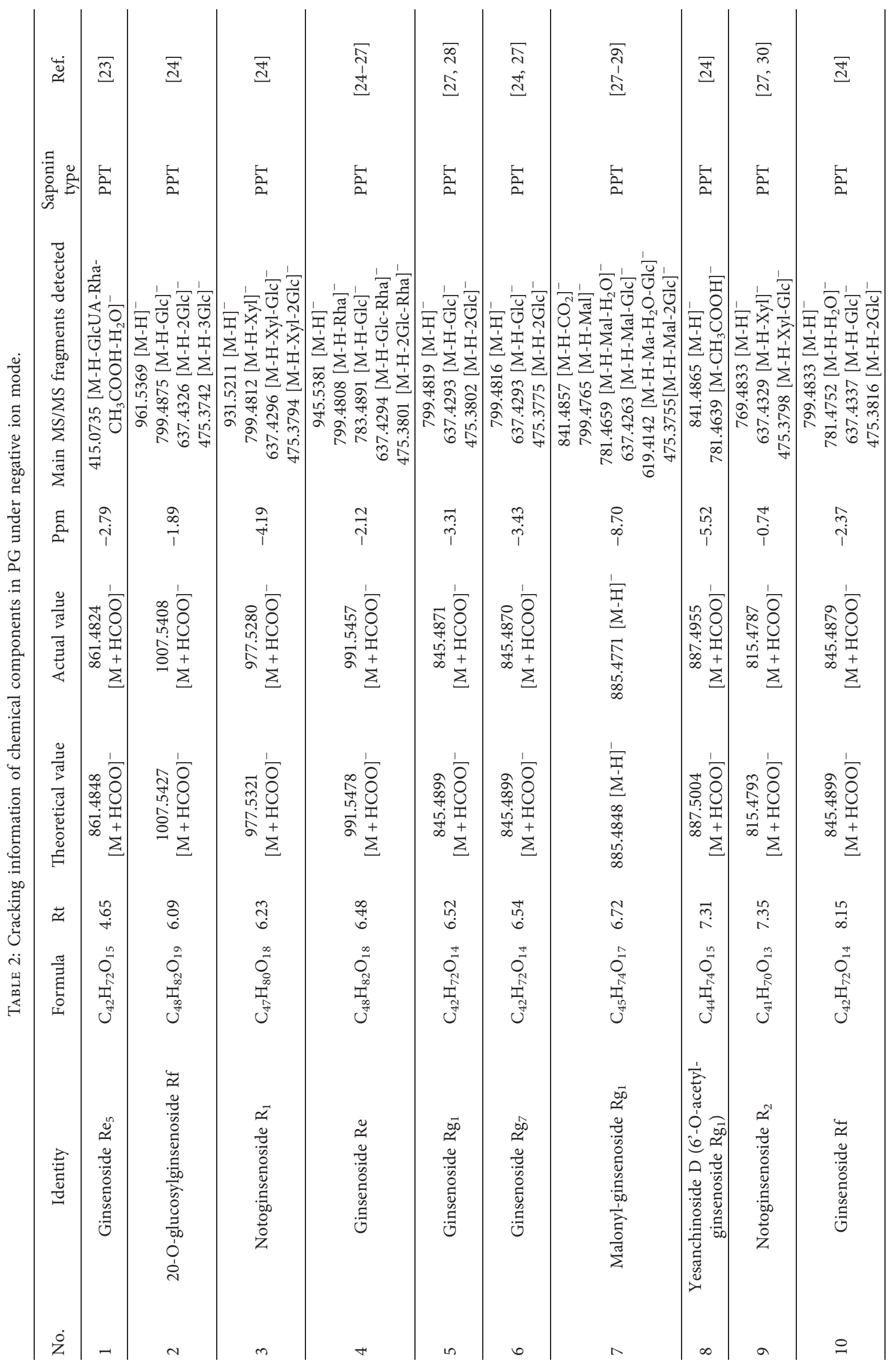




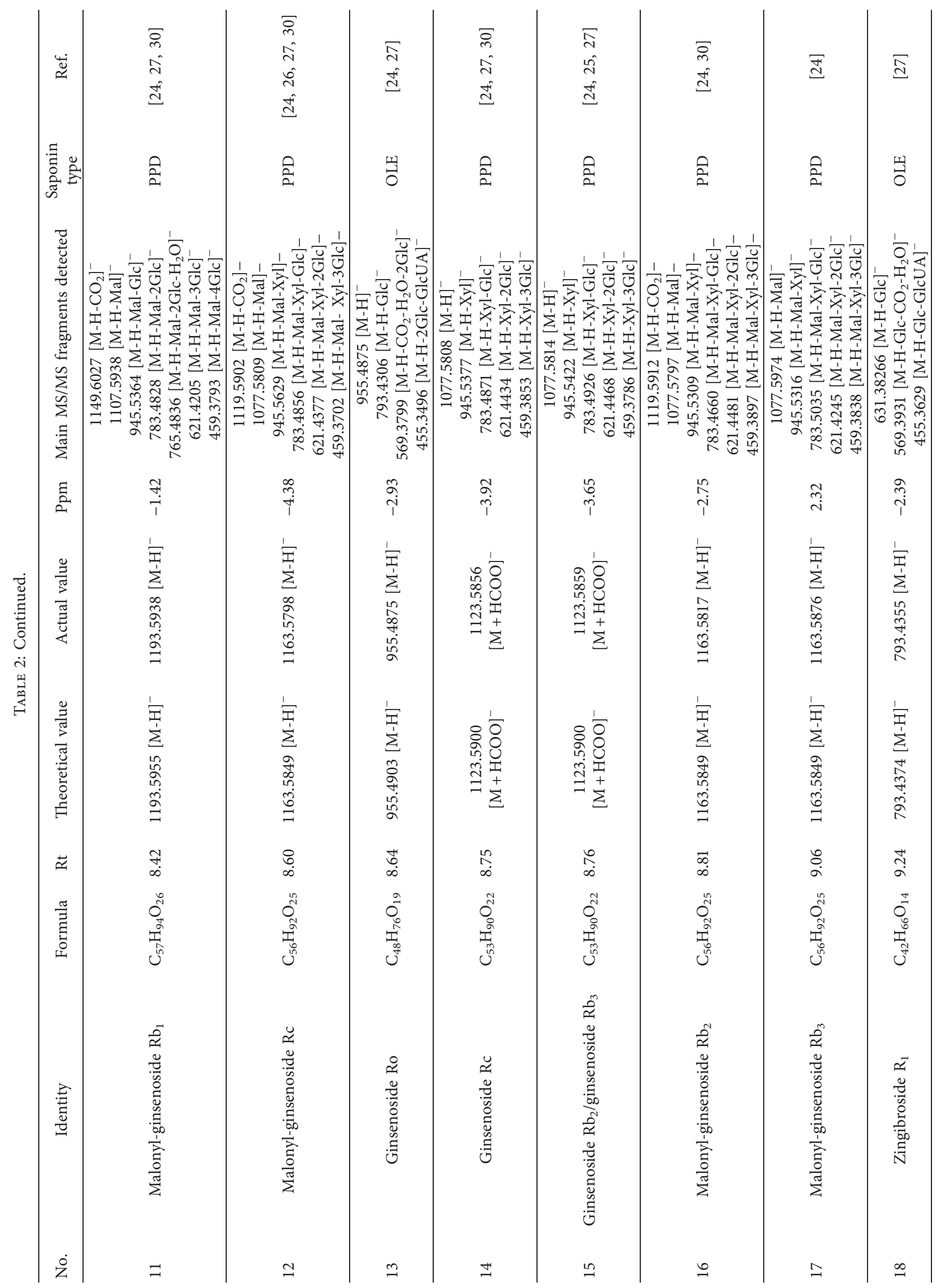




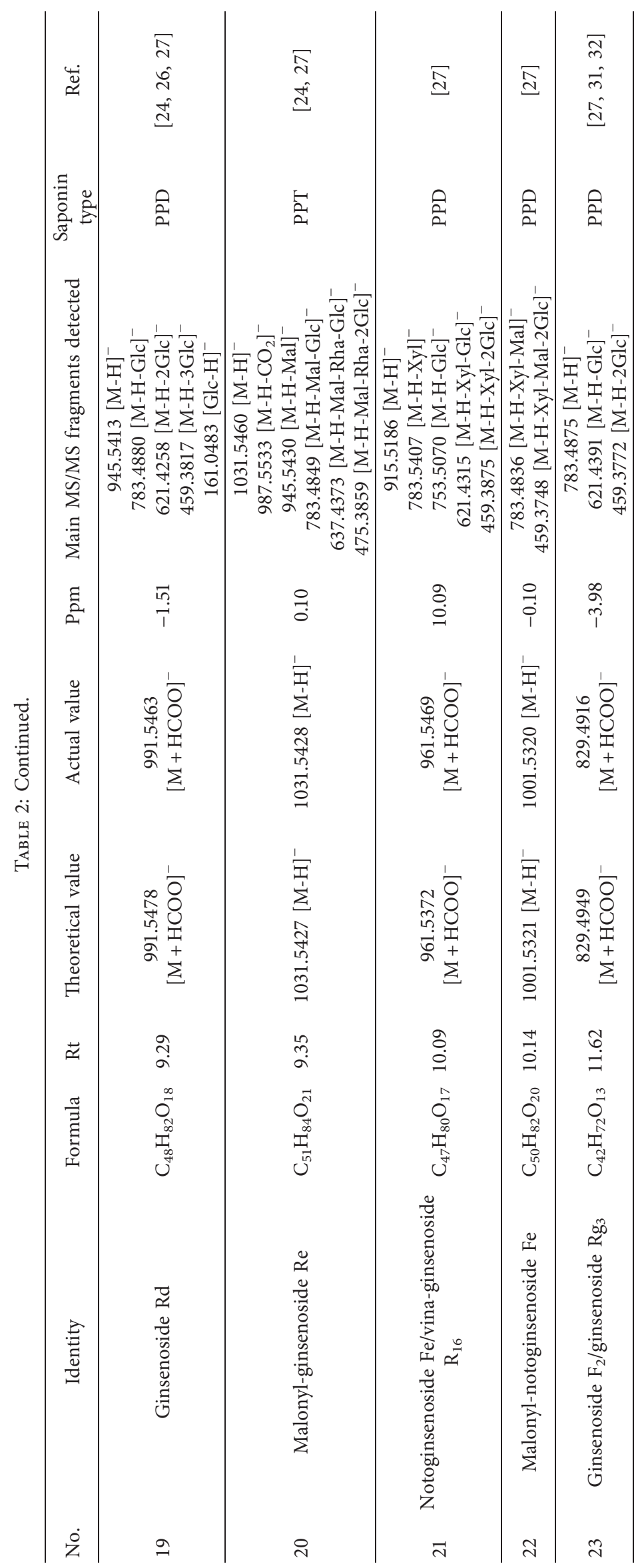




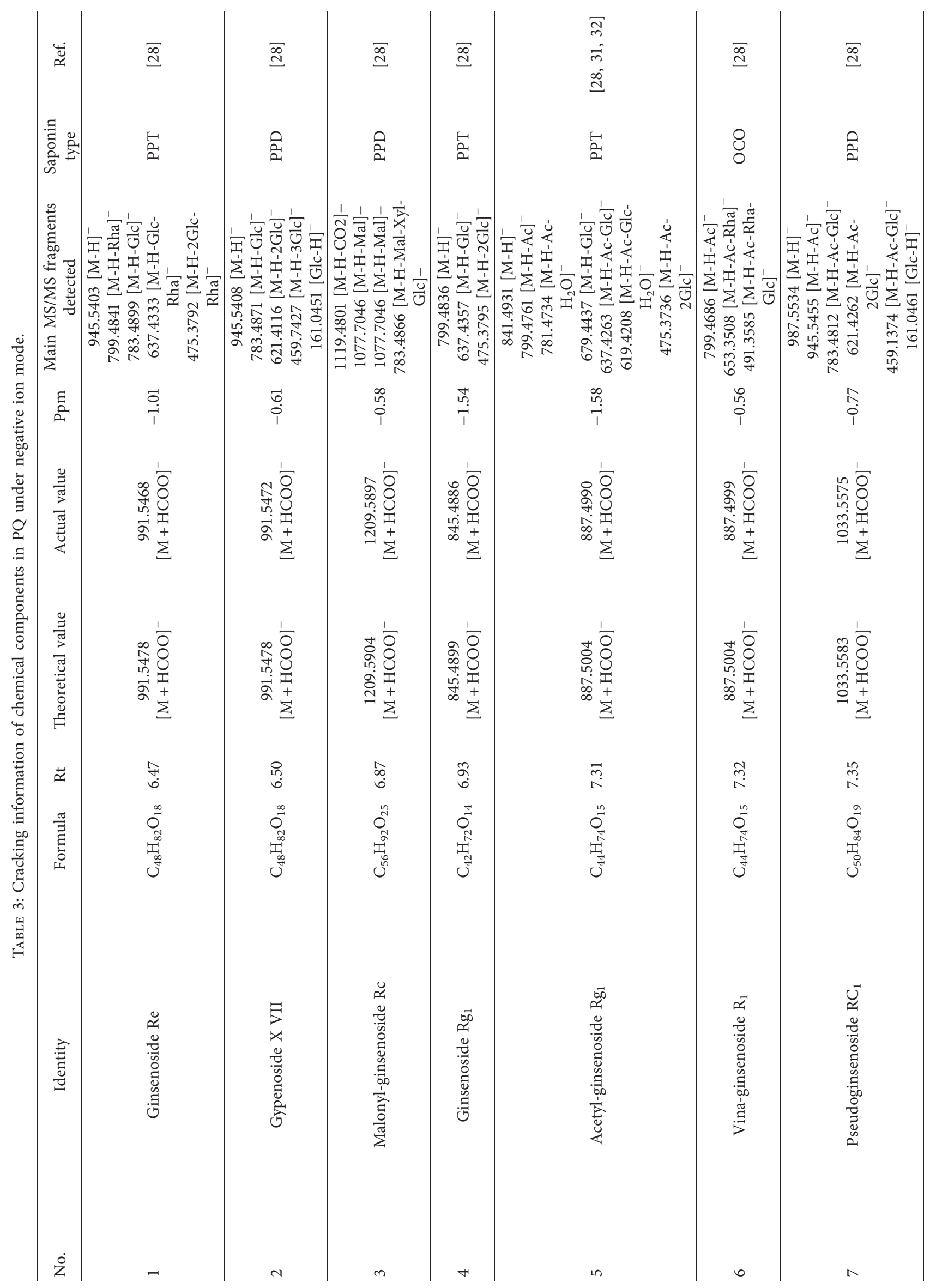




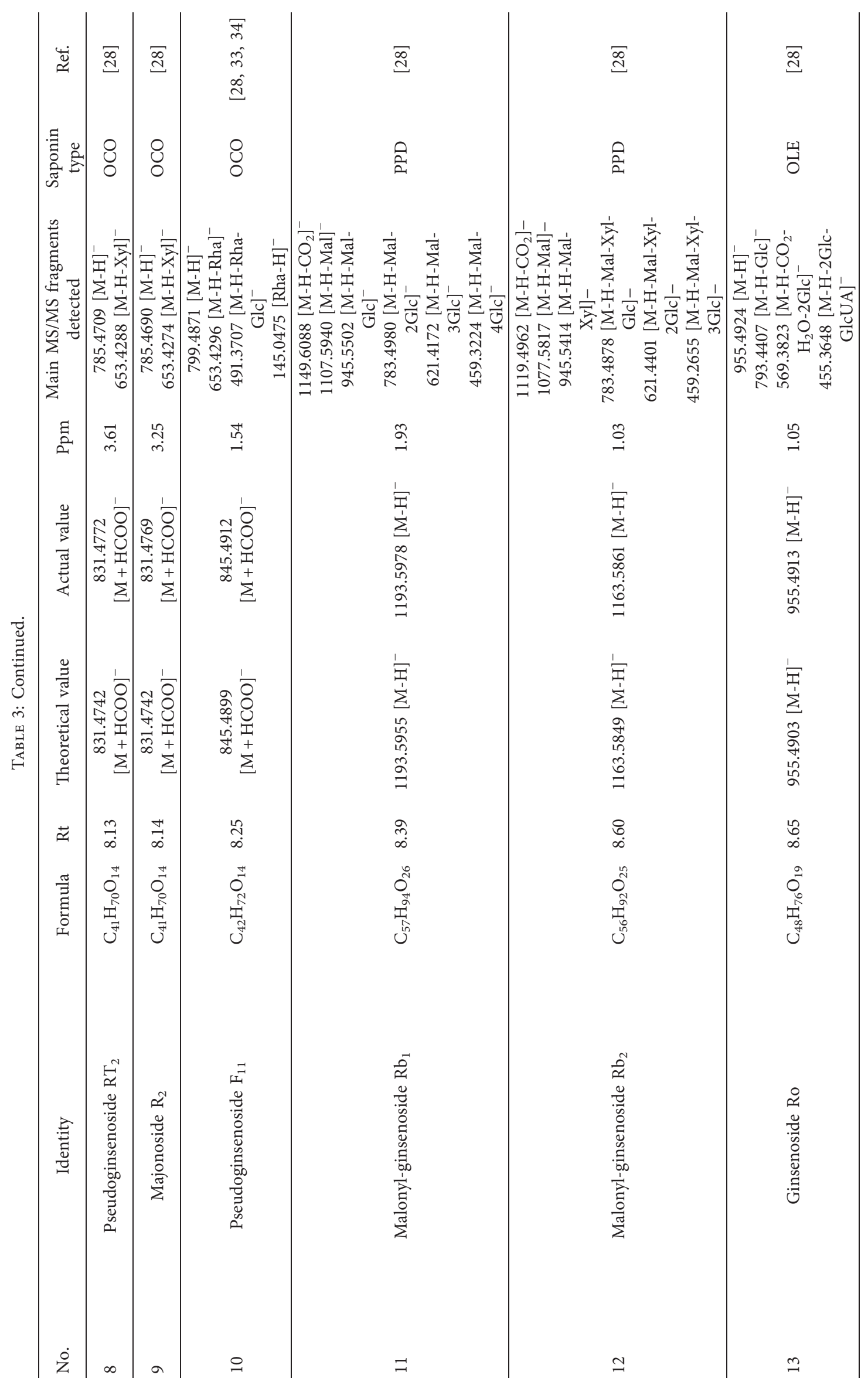




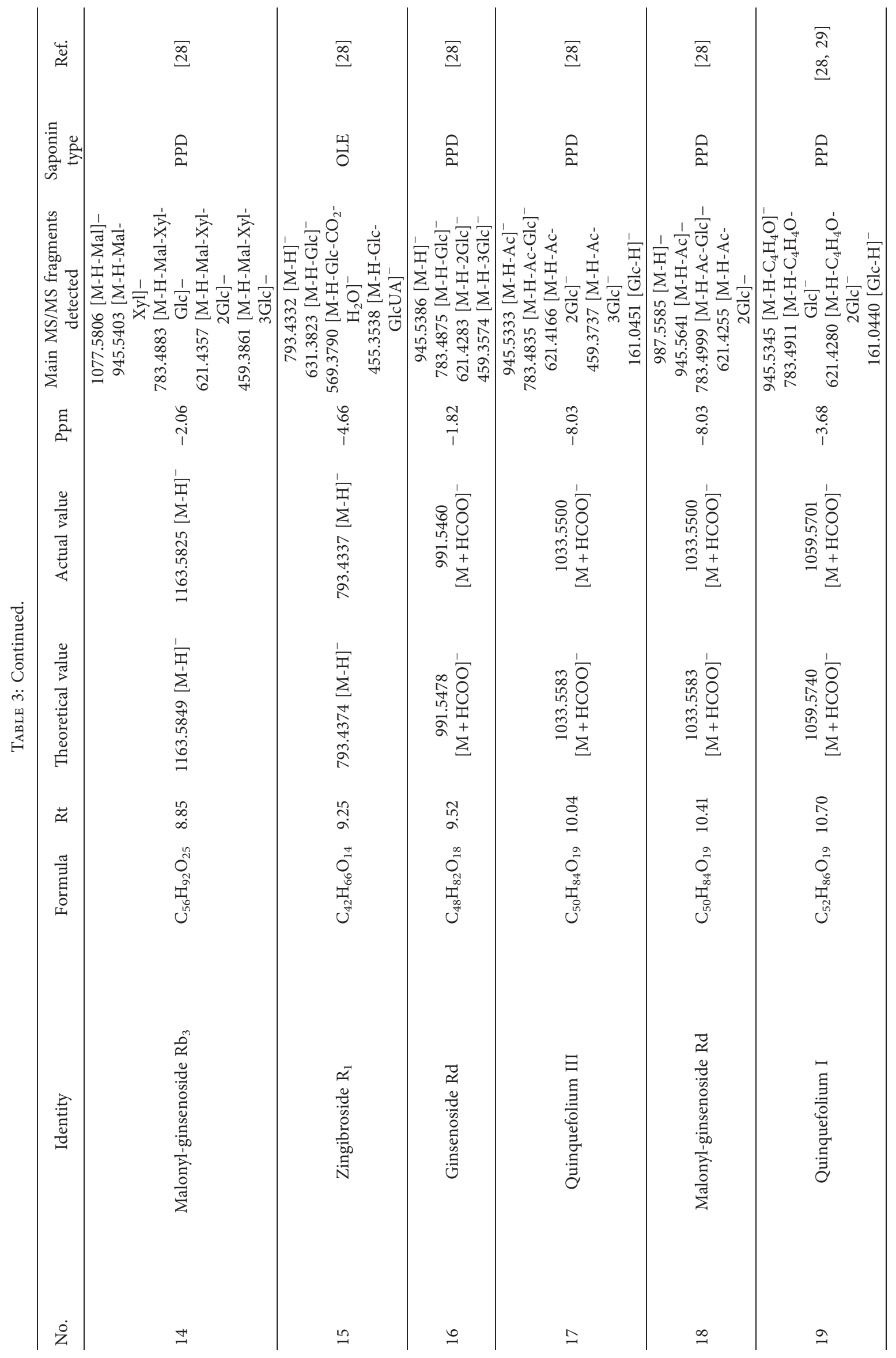




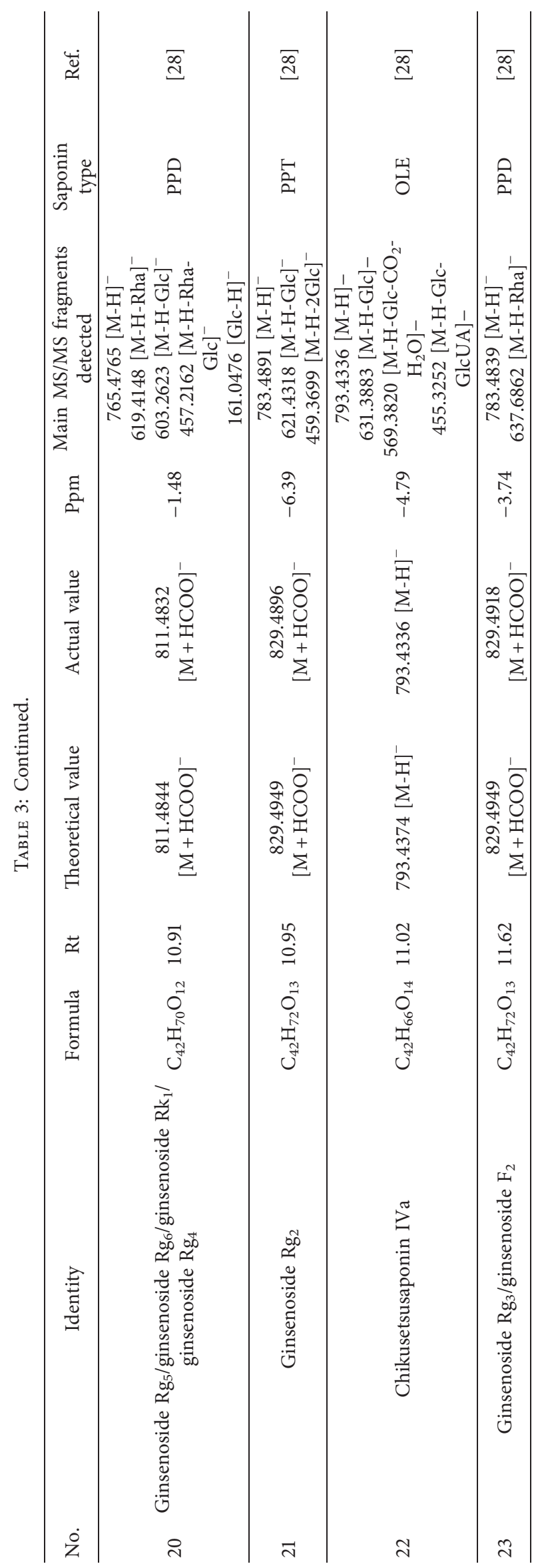




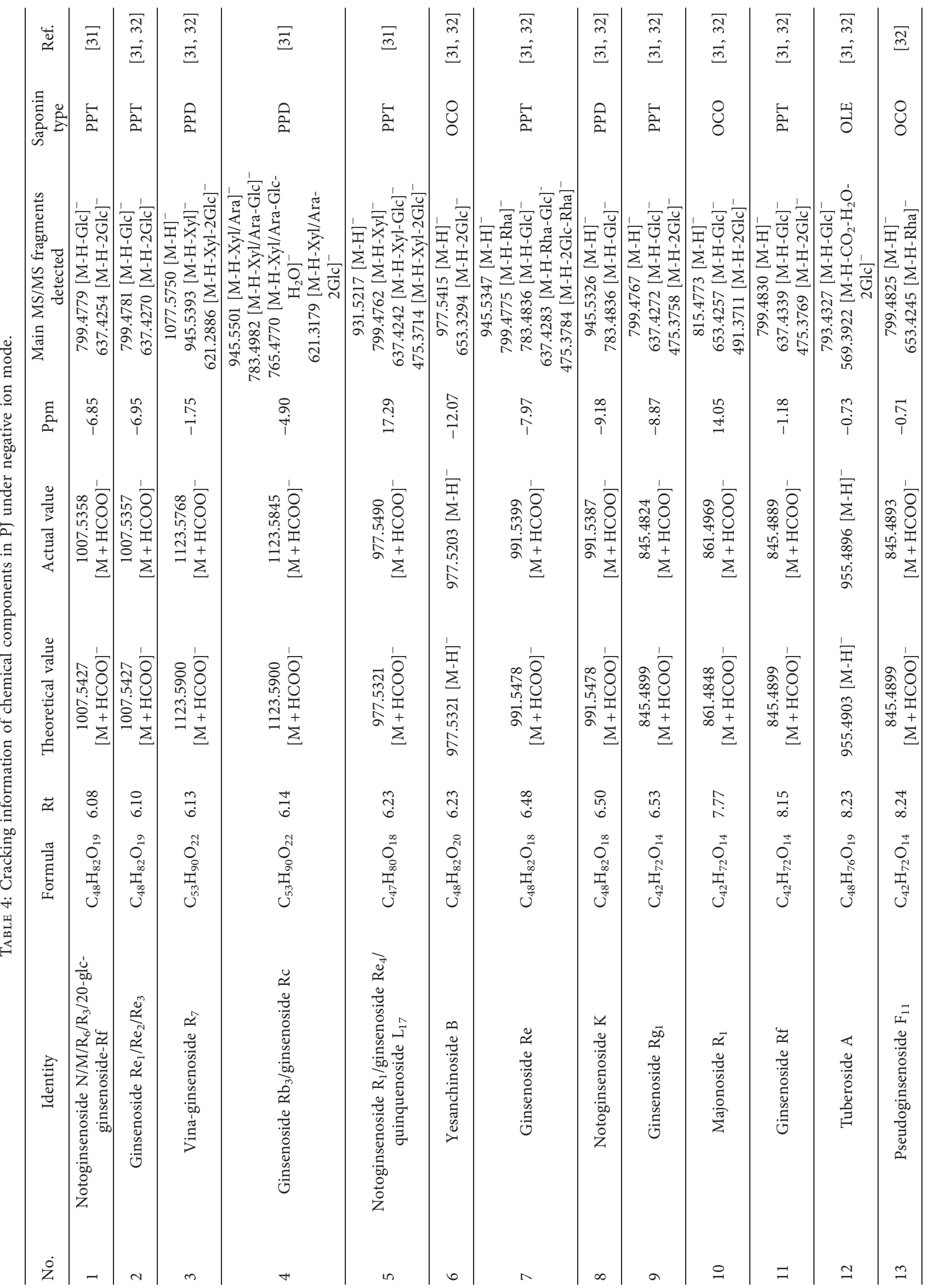




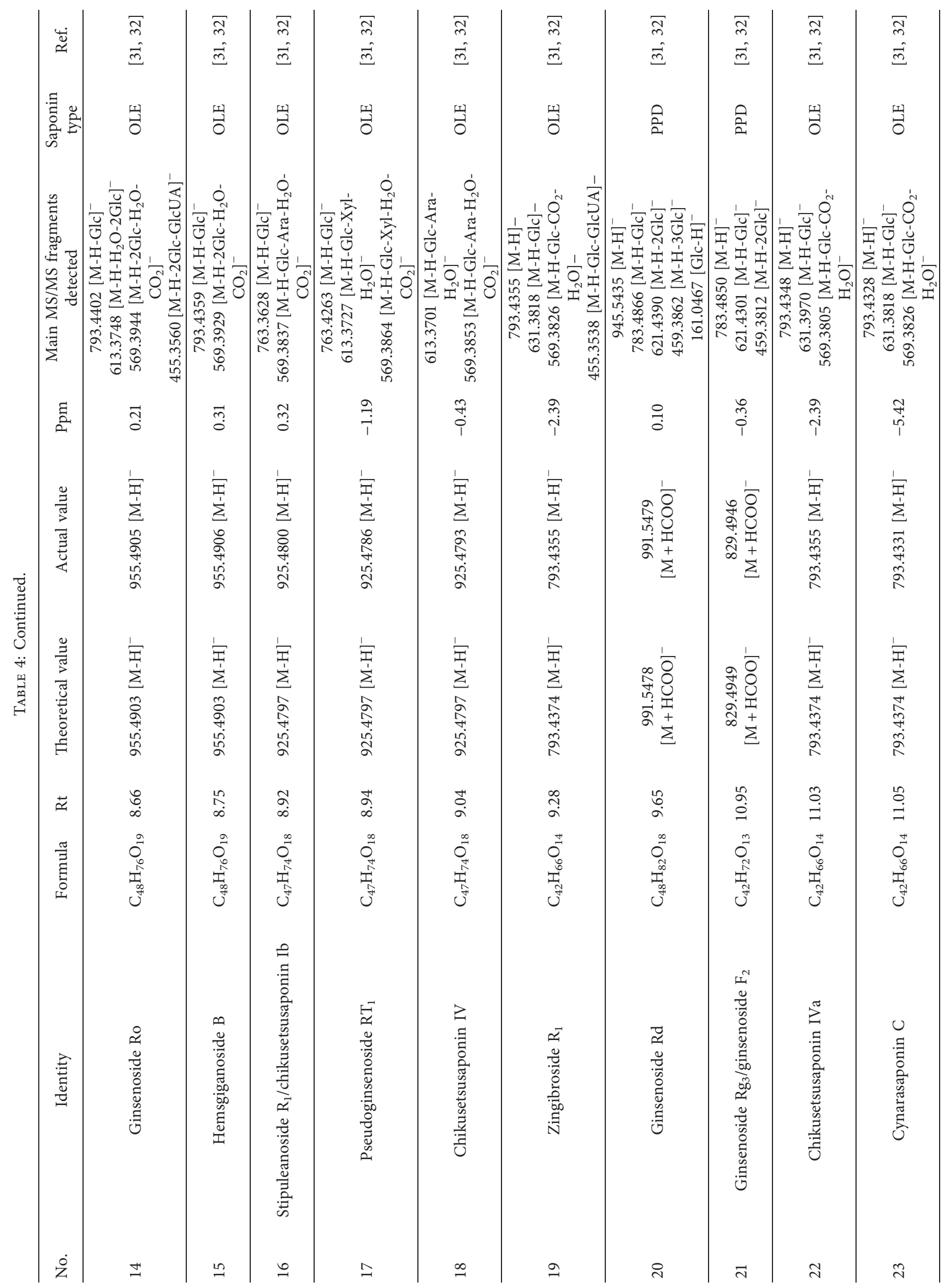




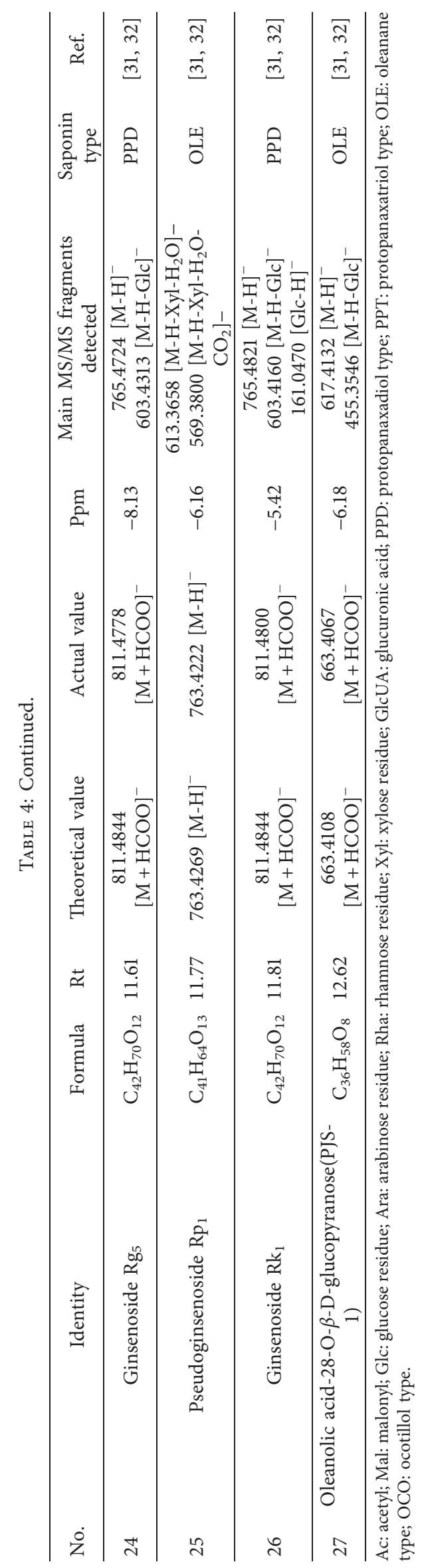




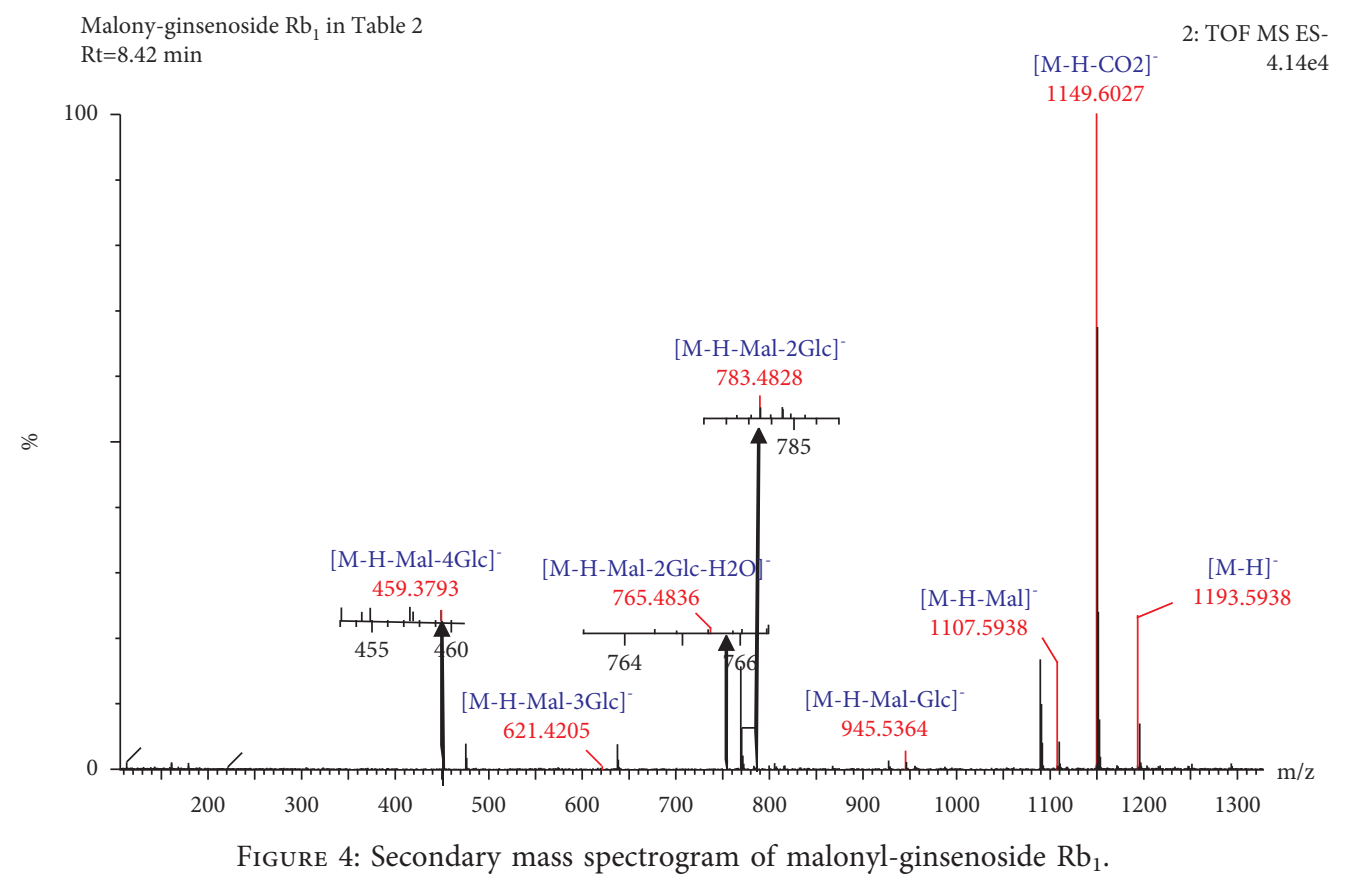

3.1.2. PPT-Type Saponins. Thus far, PPT-type saponins, such as ginsenoside $\mathrm{Re}$ and ginsenoside $\operatorname{Rg}_{1}$, have been found in ginseng plants. Notably, PQ did not contain ginsenoside Rf. The CFs of these saponins occurred at $\mathrm{m} / z$ $637\left[\mathrm{C}_{36} \mathrm{H}_{61} \mathrm{O}_{9}\right]^{-}$and $m / z 475\left[\mathrm{C}_{30} \mathrm{H}_{51} \mathrm{O}_{4}\right]^{-}$and include GlcUA (176 Da), Rha (146 Da), $\mathrm{CH}_{3} \mathrm{COOH}(60 \mathrm{Da}), \mathrm{H}_{2} \mathrm{O}$ (18 Da), $\mathrm{CO}_{2}$ (44 Da), Glc (162 Da), Xyl (132 Da), Mal $(86 \mathrm{Da})$, and Ac (42 Da). Therefore, the PPT saponins could be preliminarily identified by these product ions. This was further supported by the different NLs, which were caused by the breaking of different substituents at the C-6 and C-20 positions.

Compound 4 (Table 2) had a retention time of $6.48 \mathrm{~min}$ and a molecular formula of $\mathrm{C}_{48} \mathrm{H}_{82} \mathrm{O}_{18}$. In negative ion mode, this compound had a molecular ion peak at $\mathrm{m} / z 991.5457$ $[\mathrm{M}+\mathrm{HCOO}]^{-}$and product ion peaks at $m / z 945.5381[\mathrm{M}-\mathrm{H}]^{-}$, 799.4808 [M-H-Rha] ${ }^{-}, 783.4891$ [M-H-Glc] $^{-}, 637.4294$ [M-HGlc-Rha] $^{-}$, and 475.3801 [M-H-2Glc-Rha] ${ }^{-}$. Based on the CFs at $m / z 637.4294$ and 475.3801, the compound was identified as a PPT-type saponin. Based on the molecular ion, fragments, and reference information, compound 4 was identified as ginsenoside $\operatorname{Re}[24-27,30]$. The cleavage pathway was as follows: the molecular ion at $m / z 945.5381[\mathrm{M}-\mathrm{H}]^{-}$lost one glucose residue $(162 \mathrm{Da})$ at $\mathrm{C}-20$ to generate the product ion peak at $\mathrm{m} / z 783.4891$ [M-H-Glc] $^{-}$, while the molecular ion at $\mathrm{m} / z$ 945.5381 $[\mathrm{M}-\mathrm{H}]^{-}$lost one rhamnose residue $(146 \mathrm{Da})$ at C-6 to generate the product ion at $m / z 799.4808$ [M-H-Rha] ${ }^{-}$. When the molecular ions simultaneously lost a glucose and rhamnose residue $(162 \mathrm{Da}+146 \mathrm{Da})$, a product ion peak was produced at $m / z 637.4294$ [M-H-Glc-Rha] ${ }^{-}$. The fragment ion at $m / z 475.3801$ [M-H-2Glc-Rha] $]^{-}$was produced when the product ion at $m / z 783.4891$ lost a glucose and rhamnose residue $(162 \mathrm{Da}+146 \mathrm{Da})$ simultaneously. The fragmentation pathway of ginsenoside Re in negative-ion mode is shown in Figures 6 and 7.
Compound 11 (Table 4) had a retention time of $8.15 \mathrm{~min}$ and molecular formula of $\mathrm{C}_{42} \mathrm{H}_{72} \mathrm{O}_{14}$. In negative ion mode, compound 11 produced a precursor ion at $\mathrm{m} / z 845.4889$ $\left[\mathrm{M}+\mathrm{HCOO}^{-}\right.$and three fragment ion peaks at $m / z$ 799.4830, 637.4339 , and 475.3769. Based on the product ion peaks at $m / z$ 637.4339 and 475.3769, compound 11 was preliminarily considered to be a PPT-type saponin. When this observation was further combined with the retention time, along with the molecular ion and fragment information, compound 11 (Table 4) was identified as ginsenoside $\mathrm{Rf}[31,32]$. The cleavage pathway of ginsenoside $\mathrm{Rf}$ was as follows: the product ion peak at $m / z 637.4339$ was generated by the loss of one glucose residue $(162 \mathrm{Da})$ from the molecular ion at $m / z 799.4830$. The fragment ion peak at $m / z 475.3769$ was generated when the product ion at $m / z 637.4339$ lost one glucose residue $(162 \mathrm{Da})$. The fragmentation information and process for ginsenoside $\mathrm{Rf}$ are shown in Figures 8 and 9.

3.2. Analysis of OLE-Type Saponins by MS. Pentacyclic triterpenoid saponins of the OLE-type are characteristic components of ginseng. There are differences in the species and availability of different ginseng plants [36]. The CFs of the OLE-type saponins occurred at $\mathrm{m} / z 569\left[\mathrm{C}_{35} \mathrm{H}_{54} \mathrm{O}_{6}\right]^{-}$ and $455\left[\mathrm{C}_{30} \mathrm{H}_{47} \mathrm{O}_{3}\right]^{-}$, and the common neutral losses corresponded to GlcUA $(176 \mathrm{Da})$, Ara $(132 \mathrm{Da}), \mathrm{CO}_{2}$ (44 Da), $\mathrm{H}_{2} \mathrm{O}(18 \mathrm{Da})$, Glc $(162 \mathrm{Da})$, and $\mathrm{Xyl}(132 \mathrm{Da})$. Therefore, the OLE-type saponins could be quickly identified and described using the CF information and the retention times of the fractured C- 3 and C-28 ester bases.

Compound 13 (Table 2 ) had a retention time of $8.64 \mathrm{~min}$ and a molecular formula of $\mathrm{C}_{48} \mathrm{H}_{76} \mathrm{O}_{19}$. In negative ion mode, compound 13 was detected by the molecular ion peak at $m / z 955.4875[\mathrm{M}-\mathrm{H}]^{-}$and product ion peaks at $m / z$

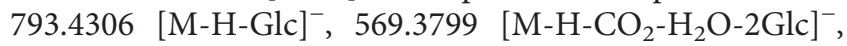




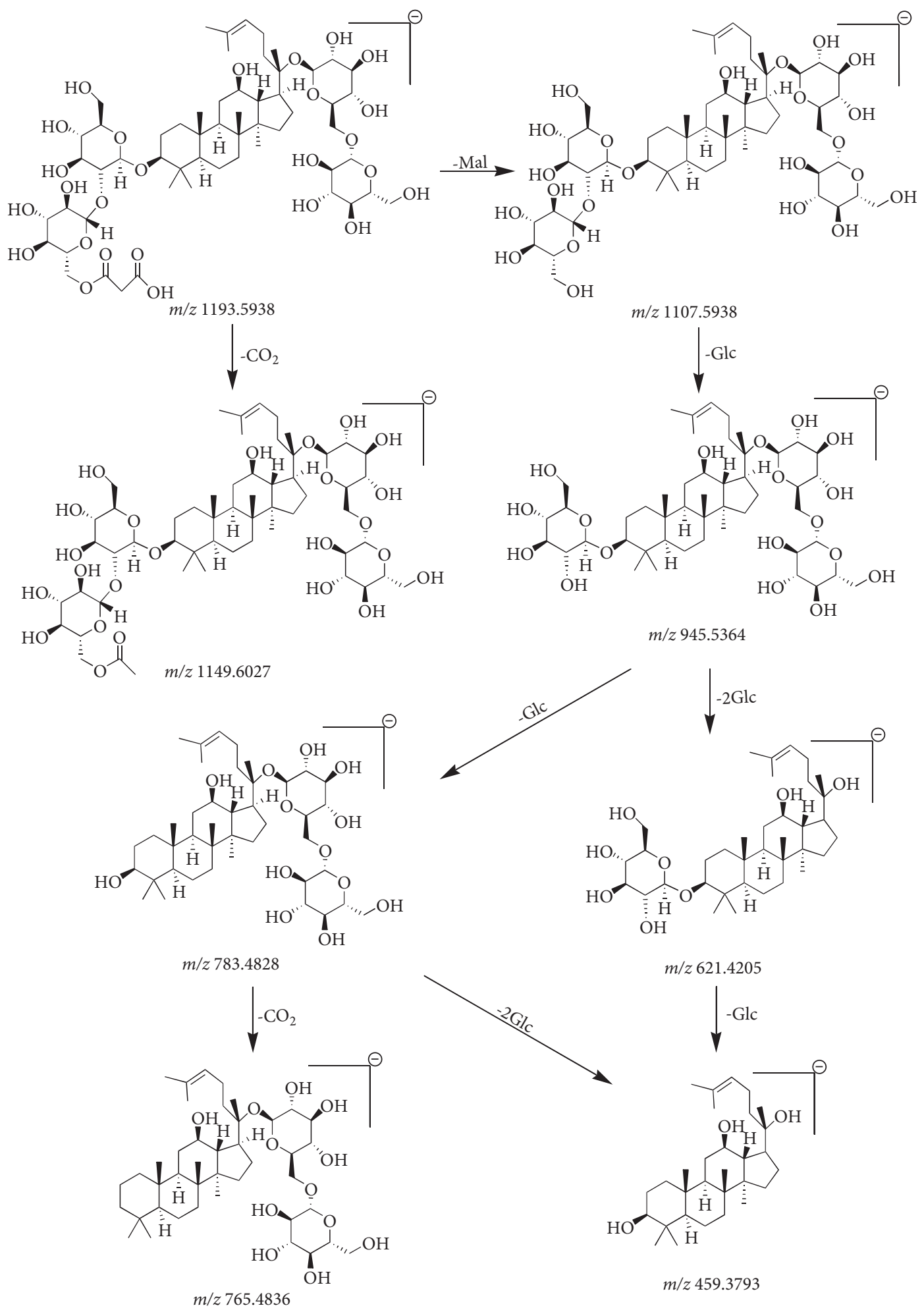

Figure 5: The fragmentation pathway of malonyl-ginsenoside $\mathrm{Rb}_{1}$.

and 455.3496 [M-H-2Glc-GlcUA $]^{-}$. Based on the CFs at $m / z$ 569.3799 and 455.3496, the compound was preliminarily determined to be an OLE-type saponin. Combining the mass spectrometry and the remaining fragment ion information (Table 2), compound 13 was identified as ginsenoside Ro [27-29]. The fragmentation of ginsenoside Ro occurred as follows: when the molecular ion at $m / z 955.4875[\mathrm{M}-\mathrm{H}]^{-}$lost one molecular glucose residue $(162 \mathrm{Da})$, fragment ion peaks were generated at $\mathrm{m} / z$ 793.4306, when the molecular ions lost one $\mathrm{CO}_{2}$ molecule (44 Da), one $\mathrm{H}_{2} \mathrm{O}(18 \mathrm{Da})$, and two molecular glucose residues $(162 \mathrm{Da}+162 \mathrm{Da})$, the product ion peak at $m / z 569.3799$ was produced. When the ions at $m /$ 


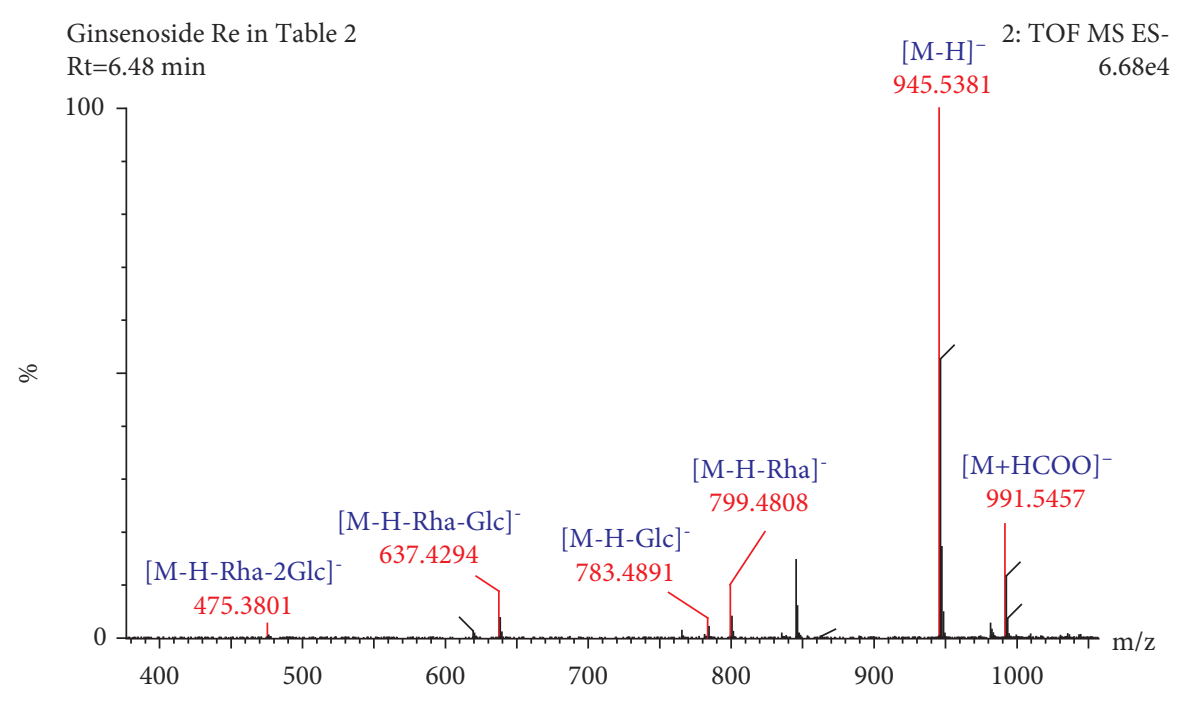

Figure 6: Secondary mass spectrogram of ginsenoside Re.

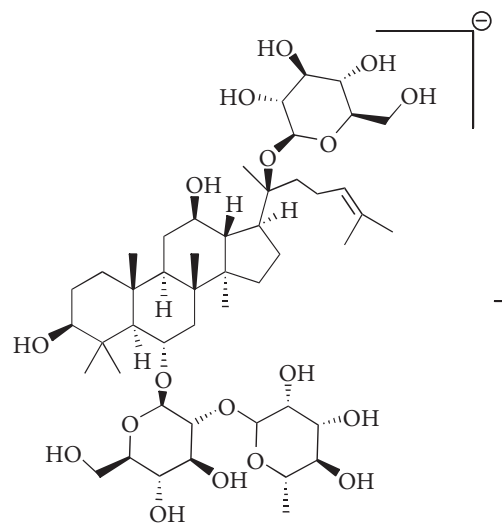
$m / z 945.5381$

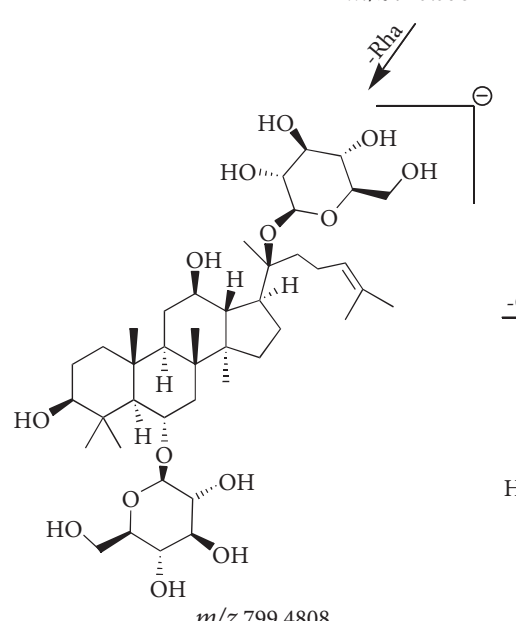

$\mathrm{m} / z 799.4808$

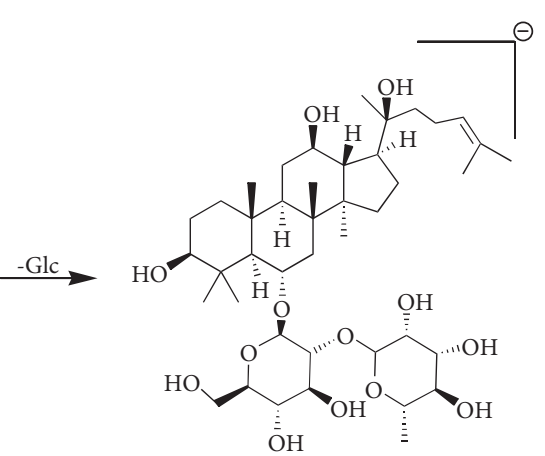

$m / z 783.4891$
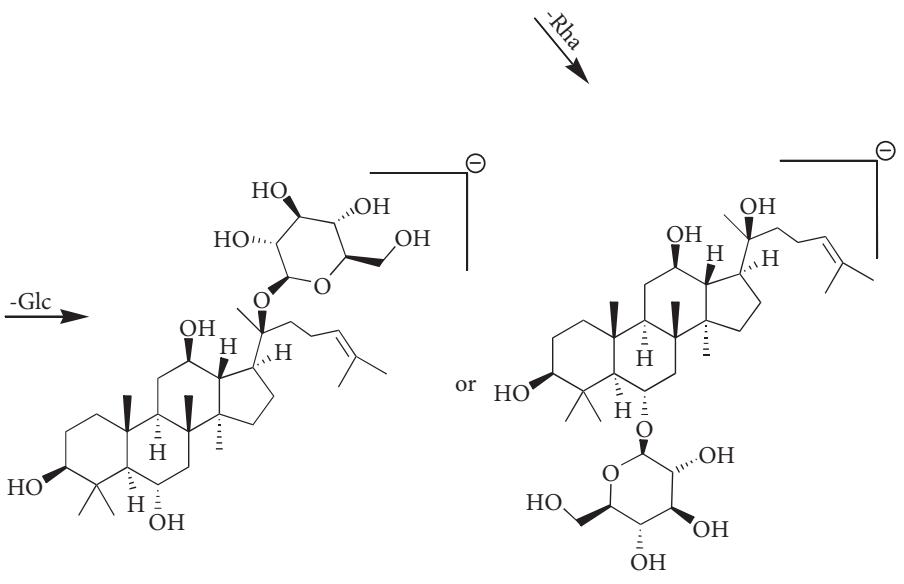

$+\sqrt{2} G_{l}$

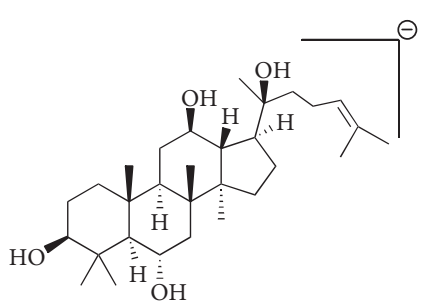

$m / z 637.4294$

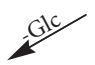

$$
\mathrm{m} / z 475.3801
$$

Figure 7: The fragmentation pathway of ginsenoside Re. 


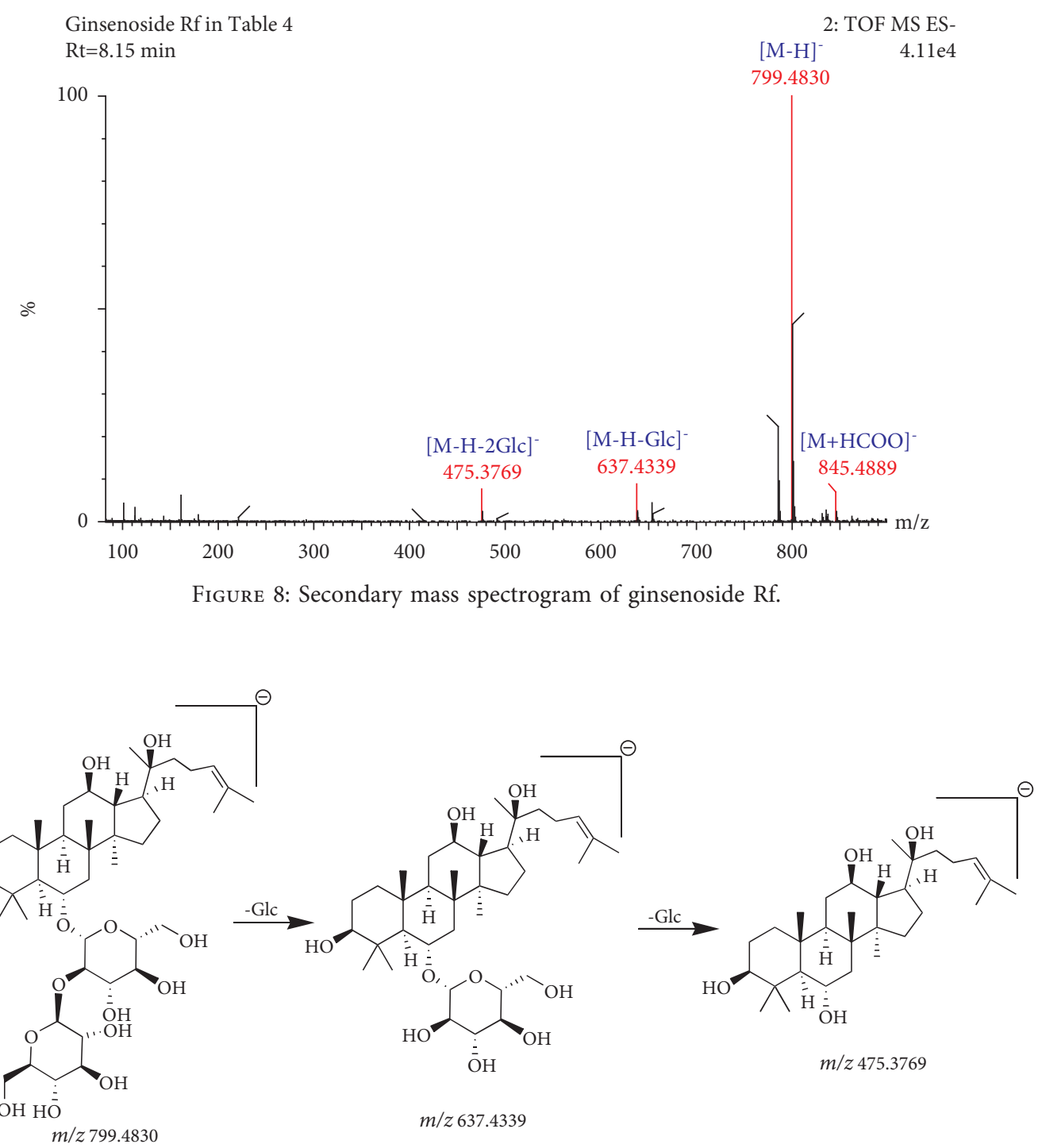

Figure 9: The fragmentation pathway of ginsenoside Rf.

z $955.4875[\mathrm{M}-\mathrm{H}]^{-}$lost two molecular glucose residues $(162 \mathrm{Da}+162 \mathrm{Da})$ and one glucuronic acid molecule $(176 \mathrm{Da})$, a resultant ion peak appeared at $\mathrm{m} / z$ 455.3496. The fragmentation of ginsenoside Ro is shown in Figures 10 and 11.

3.3. Analysis of OCO-Type Saponins by Mass Spectrometry. A furan ring was introduced into the C-20 and C-24 positions of the dammarane skeleton through a connection with oxygen, which resulted in the formation of an OCOtype saponin [15]. Studies have shown that ginseng does not contain such saponins, and as a characteristic feature, the types and contents of OCO-type saponins in PQ and PJ are also different. The results showed that the ions at $\mathrm{m} / z 653$ $\left[\mathrm{C}_{36} \mathrm{H}_{61} \mathrm{O}_{9}\right]^{-}$and $m / z 491\left[\mathrm{C}_{30} \mathrm{H}_{51} \mathrm{O}_{5}\right]^{-}$were $\mathrm{CFs}$ associated with OCO-type saponins. Ac (42 Da), Rha (146 Da), and Glc $(162 \mathrm{Da})$ were the common NL fragments. From this information, the general cleavage behavior of OCO-type saponins from PQ and PJ could be identified and proposed.
Compound 10 in Table 3, with a retention time of 8.25 min and a molecular formula of $\mathrm{C}_{42} \mathrm{H}_{72} \mathrm{O}_{14}$, generated molecular ion peaks at $m / z 845.4912[\mathrm{M}+\mathrm{HCOO}]^{-}$and fragment ion peaks at $m / z$ 799.4871, 653.4296, 491.3707, and 145.0475 in negative ion mode. Based on the CF ion peaks at $m / z 653.4296$ and $m / z$ 491.3707, the compound was identified as an OCO-type saponin. When this observation was combined with the literature data and molecular weight, the compound was identified as pseudoginsenoside $\mathrm{F}_{11}[33,34]$. The cleavage pathway is as follows: when the molecular ion at $m / z 799.4871[\mathrm{M}-\mathrm{H}]^{-}$ lost one molecular rhamnose residue $(146 \mathrm{Da})$, the product ion at $m / z 653.4296$ [M-H-Rha $]^{-}$was produced. Subsequently, the fragment ion peak at $m / z 491.3707$ [M-H-RhaGlc] ${ }^{-}$was produced by the loss of one molecular rhamnose residue and one molecular glucose residue $(146 \mathrm{Da}+162 \mathrm{Da})$. In negative ion mode, the product ion peak at $m / z 145.0475[\mathrm{Rha}-\mathrm{H}]^{-}$was examined for free rhamnose residues. The cleavage pathway of pseudoginsenoside $F_{11}$ is shown in Figures 12 and 13. 


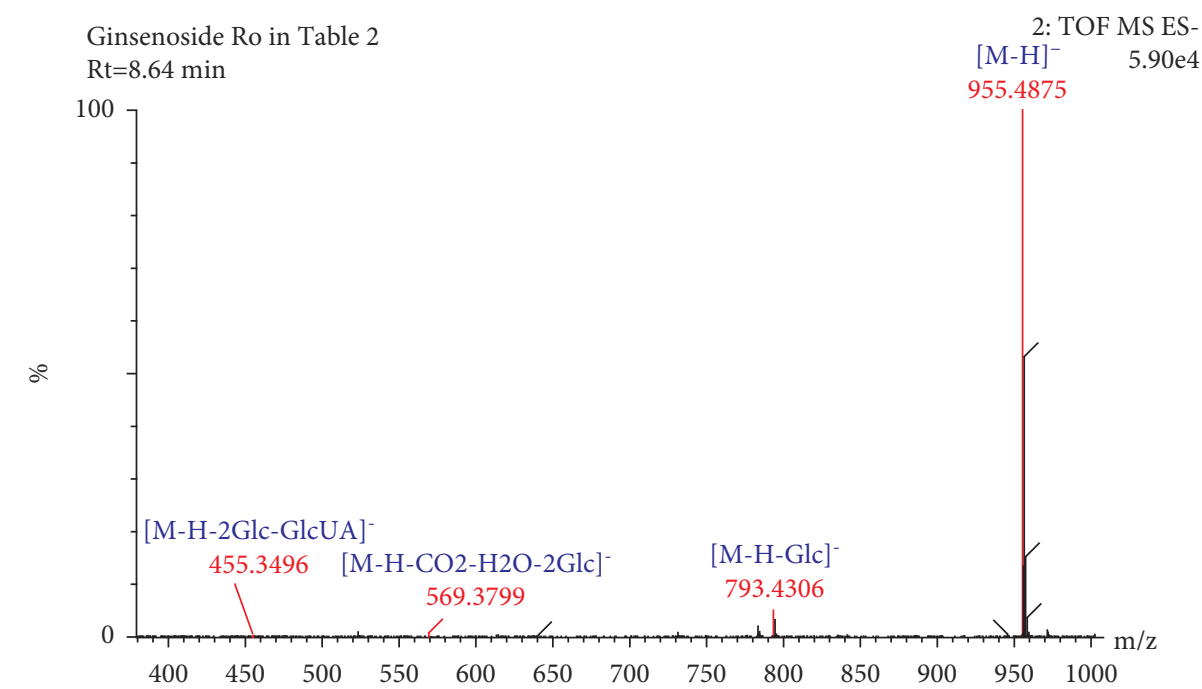

Figure 10: Secondary mass spectrogram of ginsenoside Ro.

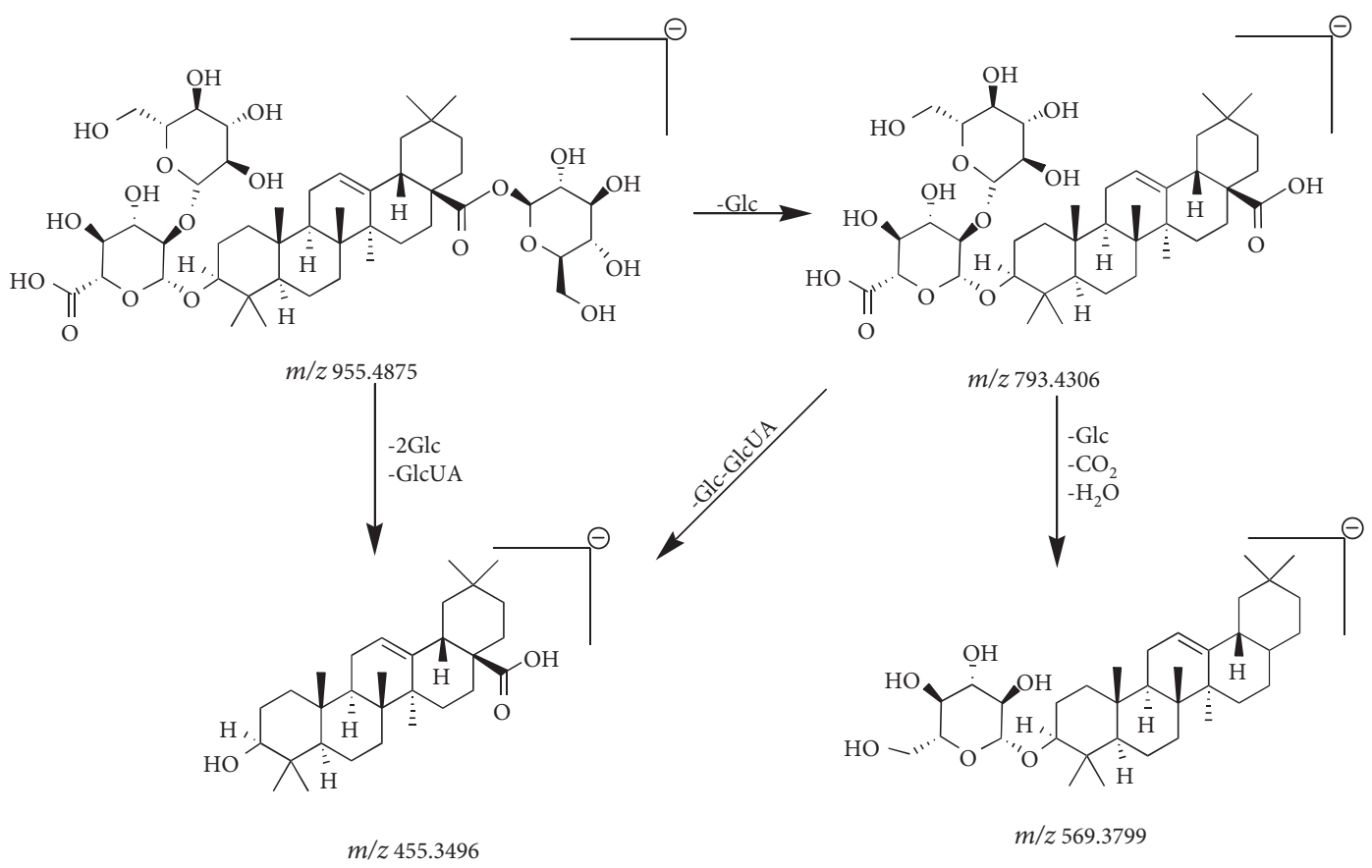

FIgURE 11: The fragmentation pathway of ginsenoside Ro.

3.4. Analysis of Differences in Saponins. From identification of the chemical components, the characteristic absence of ginsenoside Rf in PQ was noted; on this premise, PQ can be differentiated. OCO saponins were not found in PG, which could be useful in identifying PG and PJ. Based on the information in Tables 2-4, the distribution of saponins among PG, PQ, and PJ are shown in the Venn diagram in Figure 14(a). The results show that ginsenoside $\mathrm{Rg}_{1}$, zingibroside $\mathrm{R}_{1}$, ginsenoside Re, ginsenoside Ro, and ginsenoside Rd were the common components of PG, PQ, and PJ. Based on this information, the ginsenoside content in $P G, P Q$, and PJ was preliminarily analyzed. The main ginsenosides $\left(\mathrm{Rg}_{1}\right.$, $\mathrm{Re}, \mathrm{Rb}_{1}, \mathrm{Rc}$, and $\mathrm{Rd}$ ) generally account for more than $70 \%$ of the total content of ginsenosides in PQ [37]. The common components, the characteristic components of the three traditional Chinese medicines, along with ginsenoside $\mathrm{Rg}_{1}$, zingibroside $\mathrm{R}_{1}$, ginsenoside $\mathrm{Re}$, ginsenoside $\mathrm{Rd}$, ginsenoside $\mathrm{Rf}$, and OCO-type saponins (taking pseudoginsenoside $\mathrm{F}_{11}$ as an example), were selected for comparison [38, 39]. The contents of these six components in the nine batches of medicinal materials were analyzed (Figure 14(b)). These results show that the common chemical components in PG, $P Q$, and PJ were present in significantly different contents and that characteristic components only existed in specific medicinal materials. Considering that the differences in the saponins in $\mathrm{PG}, \mathrm{PQ}$, and $\mathrm{PJ}$ were preliminarily analyzed, 


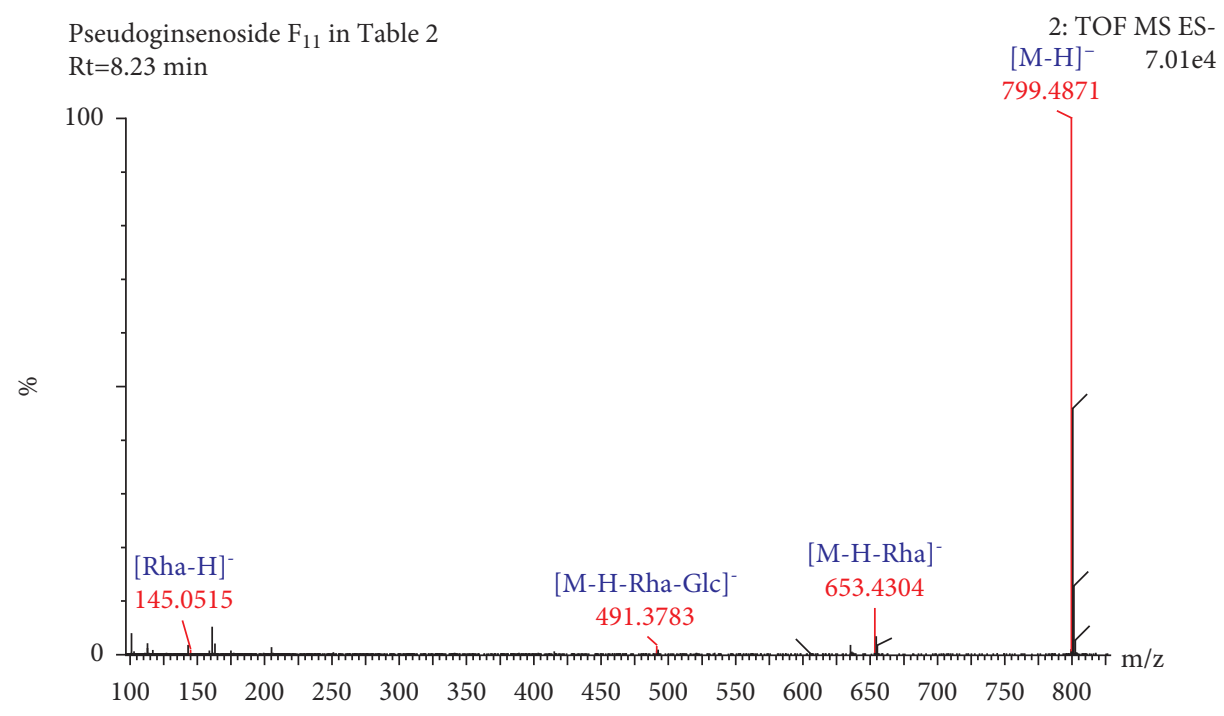

FIGURE 12: Secondary mass spectrogram of pseudoginsenoside $\mathrm{F}_{11}$.
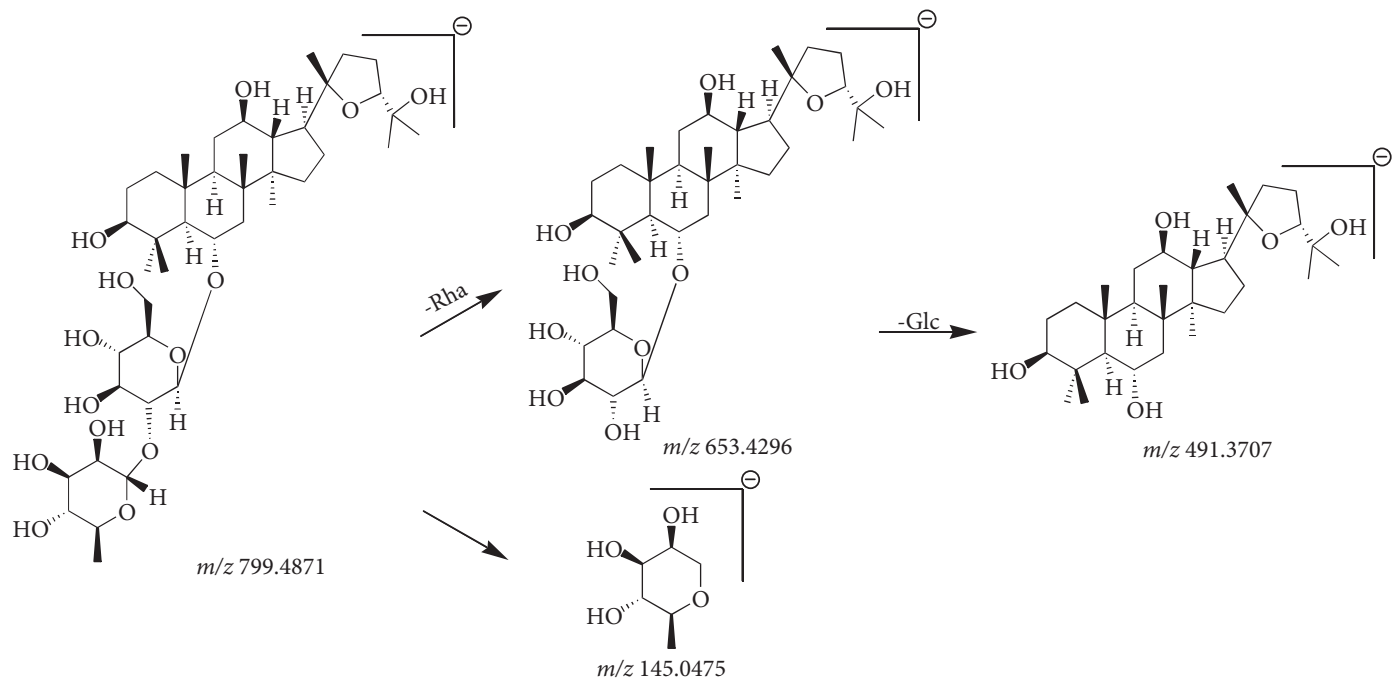

FIGURE 13: The fragmentation pathway of pseudoginsenoside $\mathrm{F}_{11}$.

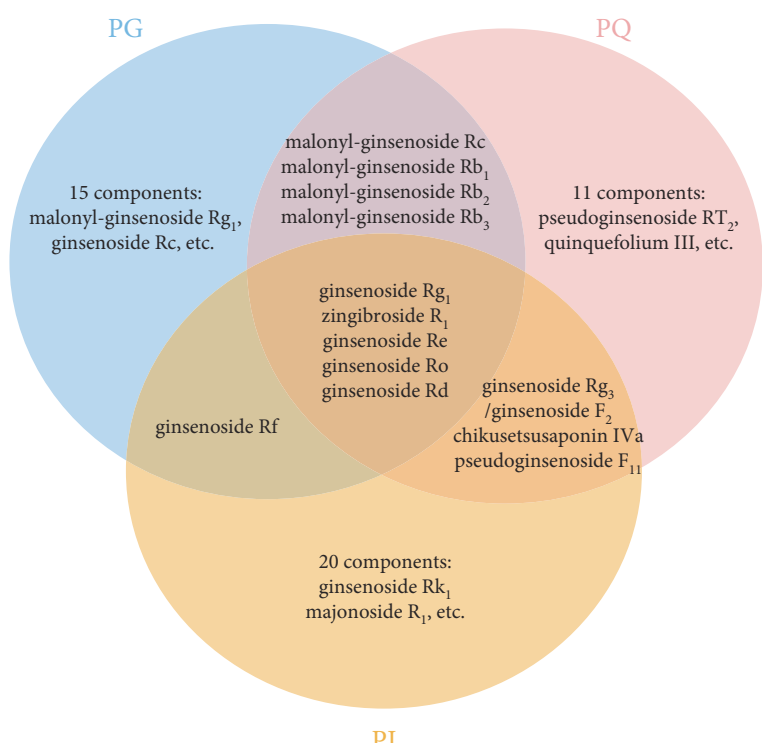

(a)

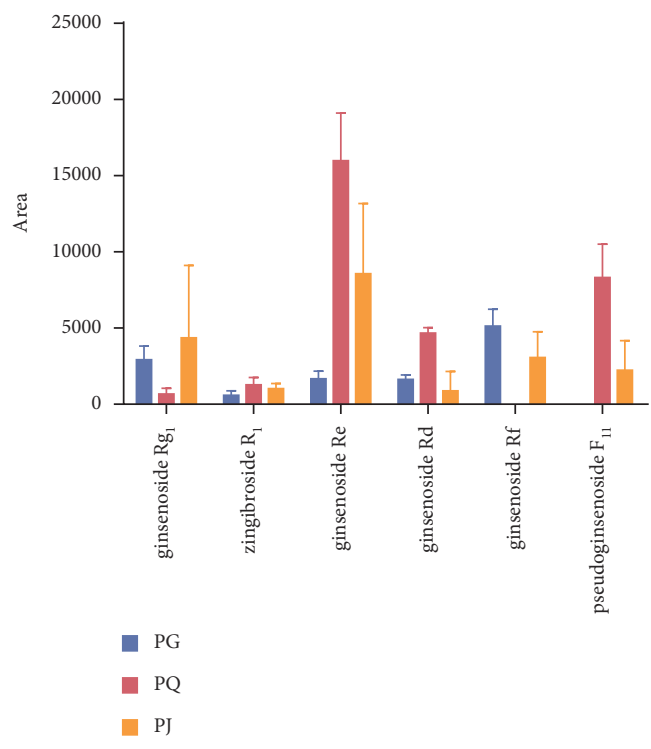

(b)

FIgURe 14: (a) Venn diagram of the distribution of saponins among PG, PQ, and PJ. (b) Contents of six components in PG, PQ, and PJ. 
future studies on the three kinds of medicinal materials from the genus Panax are needed. Nonetheless, the results have provided a foundation for the quantitative study of $P G, P Q$, and PJ and for screening the pharmacological components.

\section{Conclusions}

In this study, fragment ions associated with the chemical constituents in PG, PQ, and PJ were studied. Additionally, mass spectra fragmentation rules for the DAM-type (including PPD- and PPT-type), OLE-type, and OCO-type saponins were presented. The chemical constituents and different saponins from $\mathrm{PG}, \mathrm{PQ}$, and $\mathrm{PJ}$ were analyzed using UPLC-Q-TOF/MS. With the aid of the fragmentation rules for various components, 23 chemical components were identified in PG, 23 chemical components were identified in PQ, and 27 chemical components were identified in PJ. Among them, PG did not contain OCO-type saponins; thus, it was distinguishable from PQ and PJ. Additionally, ginsenoside Rf, a characteristic component, was not found in PQ, which provides a basis for differentiating between PQ and PJ. Through rapid classification and identification of the components, we differentiated among three types of traditional Chinese medicinal herbs from the genus Panax. This study provides a foundation for pharmacodynamic research and the development of MS in the identification of traditional Chinese medicine. Thus, this study presents a guaranteed approach for the determination of chemical components, along with the development and application of ginseng in traditional Chinese medicine.

\section{Data Availability}

No data were used to support this study.

\section{Conflicts of Interest}

The authors declare that there are no conflicts of interest with respect to the research, authorship, and/or publication of this article.

\section{Authors' Contributions}

Liu Jinbiao, Zhang Xinyue, and Yang Shenshen contributed equally to this work.

\section{Acknowledgments}

This study was supported by the National Natural Science Foundation of China (82004225 and 81903933) and the Tianjin Special Support Plan for Talent Development-HighLevel Innovation Team.

\section{References}

[1] H. Zhao, Y. He, D. Yuan, and C. Zhang, "Research advances on Panax japonicas and its approximation varieties in Tujia nationality," Agric Sci Technol, vol. 11, no. 1, pp. 126-129, 2010.
[2] T. Lou, Q. Huang, H. Su, D. Zhao, and X. Li, "Targeting sirtuin 1 signaling pathway by ginsenosides," Journal of Ethnopharmacology, vol. 268, Article ID 113657, 2021.

[3] H. Zare-Zardini, A. Alemi, and A. Taheri-Kafrani, "Assessment of a new ginsenoside Rh2 Nanoniosomal formulation for enhanced antitumor efficacy on prostate cancer: an in vitro study," Drug Design, Development and Therapy, vol. 14, pp. 3315-3324, 2020.

[4] M. Irfan, Y. S. Kwak, C. K. Han, S. H. Hyun, and M. H. Rhee, "Adaptogenic effects of Panax ginseng on modulation of cardiovascular functions," J Ginseng Res, vol. 44, no. 4, pp. 538-543, 2020.

[5] H. Liu, Y. Liu, and W. Zheng, "Extraction and separation and physiological activity of the ginsenoside from ginseng stem and leaf," Journal of Jilin Institute of Chemical Technology, vol. 31, no. 5, pp. 4-8, 2014.

[6] Y. Wang, J. Y. Pan, X. Y. Xiao, R. C. Lin, and Y. Y. Cheng, "Simultaneous determination of ginsenosides in Panax ginseng with different growth ages using high-performance liquid chromatography-mass spectrometry," Phytochemical Analysis, vol. 17, no. 6, pp. 424-430, 2006.

[7] Y. Chen, Z. Zhao, H. Chen, T. Yi, M. Qin, and Z. Liang, "Chemical differentiation and quality evaluation of commercial Asian and American ginsengs based on a UHPLCQTOF/MS/MS metabolomics approach," Phytochemical Analysis, vol. 26, no. 2, pp. 145-160, 2015.

[8] M. C. Ichim and H. J. de Boer, "A review of authenticity and authentication of commercial ginseng herbal medicines and food supplements," Frontiers in Pharmacology, vol. 11, Article ID 612071, 2020.

[9] V. B. Nguyen, H. S. Park, S. C. Lee, J. Lee, J. Y. Park, and T. J. Yang, "Authentication markers for five major Panax species developed via comparative analysis of complete chloroplast genome sequences," Journal of Agricultural and Food Chemistry, vol. 65, no. 30, pp. 6298-6306, 2017.

[10] S. Cui, J. Wu, J. Wang, and X. Wang, "Discrimination of American ginseng and Asian ginseng using electronic nose and gas chromatography-mass spectrometry coupled with chemometrics," J Ginseng Res, vol. 41, no. 1, pp. 85-95, 2017.

[11] W. R. Wu, C. S. Cheng, and Q. Q. Cheng, "Novel SNP markers on ginsenosides biosynthesis functional gene for authentication of ginseng herbs and commercial products," Chinese Journal of Natural Medicines, vol. 18, no. 10, pp. 770-778, 2020.

[12] J. Chen, L. Yang, R. Li, J. Zhang, M. Hu, and Y. Liu, "Identification of Panax japonicus and its related species or adulterants using ITS2 sequence," Chinese Traditional and Herbal Drugs, vol. 49, no. 15, pp. 3672-3680, 2018.

[13] H. Liu, X. Lu, Y. Hu, and X. Fan, "Chemical constituents of Panax ginseng and Panax notoginseng explain why they differ in therapeutic efficacy," Pharmacological Research, vol. 161, Article ID 105263, 2020.

[14] W. Z. Yang, Y. Hu, W. Y. Wu, M. Ye, and D. A. Guo, "Saponins in the genus Panax L. (Araliaceae): a systematic review of their chemical diversity," Phytochemistry, vol. 106, pp. 724, 2014.

[15] J. Liu, Y. Xu, and J. Yang, "Discovery, semisynthesis, biological activities, and metabolism of ocotillol-type saponins," J Ginseng Res, vol. 41, no. 3, pp. 373-378, 2017.

[16] W. Yang, X. Qiao, and K. Li, "Identification and differentiation of Panax ginseng, Panax quinquefolium, and Panax notoginseng by monitoring multiple diagnostic chemical markers," Acta Pharmaceutica Sinica B, vol. 6, no. 6, pp. 568-575, 2016. 
[17] S. Yang, M. Tian, and L. Yuan, "Analysis of E. rutaecarpa alkaloids constituents in vitro and in vivo by UPLC-Q-TOFMS combined with diagnostic fragment," J Anal Methods Chem, vol. 2016, Article ID 4218967, 16 pages, 2016.

[18] L. Shan, Y. Wu, L. Yuan, Y. Zhang, Y. Xu, and Y. Li, "Rapid screening of chemical constituents in Rhizoma anemarrhenae by UPLC-Q-TOF/MS combined with data postprocessing techniques," Evid Based Complement Alternat Med, vol. 2017, Article ID 4032820, 13 pages, 2017.

[19] L. Shan, N. Yang, Y. Zhao, X. Sheng, S. Yang, and Y. Li, "A rapid classification and identification method applied to the analysis of glycosides in Bupleuri radix and liquorice by ultrahigh performance liquid chromatography coupled with quadrupole time-of-flight mass spectrometry," Journal of Separation Science, vol. 41, no. 19, pp. 3791-3805, 2018.

[20] N. Yang, Y. Q. Dong, M. F. Wu, S. Z. Li, H. X. Yu, and S. S. Yang, "Establishing a rapid classification and identification method for the major triterpenoids of Alisma orientale," Phytochemical Analysis, vol. 31, no. 3, pp. 384-394, 2020.

[21] N. Li, L. Xie, and N. Yang, "Rapid classification and identification of chemical constituents in Epimedium koreanum Nakai by UPLC-Q-TOF-MS combined with data post-processing techniques," Phytochemical Analysis, vol. 32, no. 4, pp. 575-591, 2021.

[22] S. Yang, X. Zhang, Y. Dong, G. Sun, A. Jiang, and Y. Li, "Cleavage rules of mass spectrometry fragments and rapid identification of chemical components of Radix paeoniae Alba using UHPLC-Q-TOF-MS," Phytochemical Analysis, vol. 23, pp. 1-14, 2021.

[23] H. P. Wang, X. B. Yang, and X. W. Yang, "Ginsenjilinol, a new protopanaxatriol-type saponin with inhibitory activity on LPS-activated NO production in macrophage RAW 264.7 cells from the roots and rhizomes of Panax ginseng," Journal of Asian Natural Products Research, vol. 15, no. 5, pp. 579-587, 2013.

[24] Y. Yang, Y. Yang, and H. Qiu, "Localization of constituents for determining the age and parts of ginseng through ultraperfomance liquid chromatography quadrupole/time of flight-mass spectrometry combined with desorption electrospray ionization mass spectrometry imaging," Journal of Pharmacy Biomedicine Analytical, vol. 193, Article ID 113722, 2021.

[25] J. Liu, P. Chen, L. Chen, Q. Wang, H. Wu, and R. Xu, "Analysis on chemical components of Shenqi guishao decoction by UPLC-Q-TOF/MS," Chin J Pharm Anal, vol. 36, no. 9, pp. 1578-1584, 2016.

[26] G. Jin, J. Sun, and H. Zhang, "Identifying ginsenosides in ginseng whitening cream using electrospray ionization tandem mass spectrometry," Modernization of traditional Chinese medicine and materia medical-world science and technology, vol. 11, no. 3, pp. 413-415, 2009.

[27] Y. L. Guo, Y. Wang, and Y. L. Zhao, "Chemical comparison of white ginseng before and after extrusion by UHPLC-QOrbitrap-MS/MS and multivariate statistical analysis," J Anal Methods Chem, vol. 2020, Article ID 4764219, 23 pages, 2020.

[28] X. Huang, Y. Liu, and Y. Zhang, "Multicomponent assessment and ginsenoside conversions of Panax quinquefolium L. roots before and after steaming by HPLC-MSn," J Ginseng Res, vol. 43, no. 1, pp. 27-37, 2019.

[29] L. Li, L. Tan, and C. Wang, "Identification of chemical constituents of American ginseng fruit pedicels by ultraperformance liquid chromatography quadrupole time-of- flight mass spectrometry," Chinese Journal of Applied Chemistry, vol. 38, no. 3, pp. 256-270, 2021.

[30] L. Zhao, C. Jiao, and H. Li, "Chemical transformation of protopanaxadiol type ginsenoside $\mathrm{Rb}_{1}, \mathrm{Rb}_{2}$ and $\mathrm{Rc}$ analyzed by RRLC-Q-TOF-MS," Chemical Journal of Chinese Universities, vol. 39, no. 4, pp. 667-673, 2018.

[31] J. Chen, M. Tan, and L. Zou, "Saponins in Panacis japonici rhizoma as analyzed by UFLC-Triple TOF MS/MS," Food Science, vol. 40, no. 14, pp. 249-258, 2019.

[32] J. Chen, M. Tan, and L. Zou, "Qualitative and quantitative analysis of the saponins in Panacis japonici rhizoma using ultra-fast liquid chromatography coupled with triple quadrupole-time of flight tandem mass spectrometry and ultra-fast liquid chromatography coupled with triple quadrupole-linear ion trap tandem mass spectrometry," Chemical \& Pharmaceutical Bulletin, vol. 67, no. 8, pp. 839-848, 2019.

[33] J. P. Liu, F. Wang, P. Y. Li, and D. Lu, “A new ocotillol-type triterpenoid saponin from red American ginseng," Natural Product Research, vol. 26, no. 8, pp. 731-735, 2012.

[34] L. Li, G. A. Luo, Q. L. Liang, P. Hu, and Y. M. Wang, "Rapid qualitative and quantitative analyses of Asian ginseng in adulterated American ginseng preparations by UPLC/QTOF-MS," Journal of Pharmacy Biomedicine Analytical, vol. 52, no. 1, pp. 66-72, 2010.

[35] S. Shibata, O. Tanaka, T. Ando, M. Sado, S. Tsushima, and T. Ohsawa, "Chemical studies on oriental plant drugs. XIV. Protopanaxadiol, a genuine sapogenin of ginseng saponins," Chemical \& Pharmaceutical Bulletin, vol. 14, no. 6, pp. 595600, 1966.

[36] Q. Wu, P. Chen, and Q. Zhang, "Advances in research of chemical constituents, pharmacological activities and analytical methods of Panax japonicus," Asia Pac Trad Med, vol. 12, no. 6, pp. 46-54, 2016.

[37] J. Yu, T. Xu, H. Lin, Y. Lin, J. Zhou, and Y. Zhang, "Comprehensive quality evaluation of American ginseng for different parts and abnormal trait based on the major ginsenoside contents and morphological characteristics," BioMed Research International, vol. 2021, Article ID 8831080, 8 pages, 2021.

[38] X. Wang, Q. Xie, Y. Liu et al., "Panax japonicus and chikusetsusaponins: A review of diverse biological activities and pharmacology mechanism," Chinese Herbal Medicines, vol. 13, no. 1, pp. 64-77, 2021.

[39] S. Han, S. Shi, Y. Zou et al., "Chemical constituents from acid hydrolyzates of Panax quinquefolius total saponins and their inhibition activity to $\alpha$-glycosidase and protein tyrosine phosphatase 1B," Chinese Herbal Medicines, vol. 12, no. 2, pp. 195-199, 2020. 